\title{
Ionization and scintillation of nuclear recoils in gaseous xenon
}

J. Renner ${ }^{\mathrm{a}, \mathrm{b}, *}$, V. M. Gehman ${ }^{\mathrm{a}}$, A. Goldschmidt ${ }^{\mathrm{a}}$, H. S. Matis ${ }^{\mathrm{a}}$, T. Miller ${ }^{\mathrm{a}}$, Y. Nakajima ${ }^{\mathrm{a}}$, D. Nygren ${ }^{\mathrm{a}}$, C. A. B. Oliveira ${ }^{a}$, D. Shuman ${ }^{\mathrm{a}}$, V. Álvarez ${ }^{\mathrm{c}}$, F. I. G. Borges ${ }^{\mathrm{d}}, \mathrm{S}_{\text {. Cárcel }}^{\mathrm{c}}$, J. Castel $^{\mathrm{e}}, \mathrm{S}$. Cebrián ${ }^{\mathrm{e}}$, A. Cervera ${ }^{\mathrm{c}}$, C. A. N. Conde ${ }^{\mathrm{d}}$, T. Dafni ${ }^{\mathrm{e}}$, T. H. V. T. Dias ${ }^{\mathrm{d}}$, J. Díaz ${ }^{\mathrm{c}}$, R. Esteve ${ }^{\mathrm{f}}$, P. Evtoukhovitch ${ }^{\mathrm{g}}$, L. M. P. Fernandes ${ }^{\mathrm{d}}$, P. Ferrario ${ }^{\text {c }}$, A. L. Ferreira ${ }^{\text {f }}$, E. D. C. Freitas ${ }^{\text {d }}$, A. Gil ${ }^{\mathrm{c}}$, H. Gómez ${ }^{\mathrm{e}}$, J. J. Gómez-Cadenas ${ }^{\mathrm{c}, 1}$, D. González-Díaz ${ }^{\mathrm{e}}$, R. M. Gutiérrez ${ }^{\text {, J. Jauptman }}{ }^{\mathrm{i}}$, J. A. Hernando Morata ${ }^{\mathrm{j}}$, D. C. Herrera ${ }^{\mathrm{e}}$, F. J. Iguaz $^{\mathrm{e}}$, I. G. Irastorza ${ }^{\mathrm{e}}$, M. A. Jinete ${ }^{\mathrm{h}}$, L. Labarga ${ }^{\mathrm{k}}$, A. Laing ${ }^{\mathrm{c}}$, I. Liubarskyc ${ }^{\mathrm{c}}$ J. A. M. Lopes ${ }^{\mathrm{d}}$, D. Lorca ${ }^{\mathrm{c}}$, M. Losada ${ }^{\mathrm{h}}$, G. Luzón ${ }^{\mathrm{e}}$, A. Marîf ${ }^{\mathrm{f}}$ J. Martín-Albo ${ }^{\mathrm{c}}$, A. Martínez ${ }^{\mathrm{c}}$, A. Moiseenko ${ }^{\mathrm{g}}$, F. Monrabal $^{\mathrm{c}}$, M. Monserrate $^{\mathrm{c}}$, C. M. B. Monteiro ${ }^{\mathrm{d}}$, F. J. Mora ${ }^{\mathrm{f}}$, L. M. Moutinho ${ }^{1}$, J. Muñoz Vidal ${ }^{\mathrm{c}}$, H. Natal da Luz ${ }^{\mathrm{d}}$, G. Navarro ${ }^{\mathrm{h}}$, M. Nebot-Guinot ${ }^{\mathrm{c}}$, R. Palma ${ }^{\mathrm{n}}, \mathrm{J}_{\text {. Pérez }}^{\mathrm{m}}$, J. L. Pérez Aparicio ${ }^{\mathrm{n}}$, L. Ripoll ${ }^{\circ}$, A. Rodríguez ${ }^{\mathrm{e}}$, J. Rodríguez ${ }^{\mathrm{c}}$, F. P. Santos ${ }^{\text {d, J. M. F. dos Santos }}{ }^{\text {d }}$, L. Seguíe, L. Serra ${ }^{\mathrm{c}}$, A. Simón ${ }^{\mathrm{c}}$, C. Sofka ${ }^{\mathrm{p}}$, M. Sorel ${ }^{\mathrm{c}}$, J. F. Toledo ${ }^{\mathrm{f}}$, A. Tomás ${ }^{\mathrm{e}}$, J. Torrent $^{\mathrm{o}}$, Z. Tsamalaidze ${ }^{\mathrm{g}}$, J. F. C. A. Veloso ${ }^{1}$, J. A. Villar ${ }^{\mathrm{e}}$, R. C. Webb ${ }^{\mathrm{p}}$, J. White ${ }^{\mathrm{p}, 2}$, N. Yahlalic ${ }^{\mathrm{c}}$, (NEXT Collaboration)

${ }^{a}$ Lawrence Berkeley National Laboratory (LBNL), 1 Cyclotron Road, Berkeley, California 94720, USA

${ }^{b}$ Department of Physics, University of California, Berkeley, CA 94720, USA

${ }^{c}$ Instituto de Física Corpuscular (IFIC), CSIC E Universitat de València, Calle Catedrático José Beltrán, 2, 46980 Paterna, Valencia, Spain ${ }^{d}$ Departamento de Fisica, Universidade de Coimbra, Rua Larga, 3004-516 Coimbra, Portugal

${ }^{e}$ Laboratorio de Física Nuclear y Astropartículas, Universidad de Zaragoza, Calle Pedro Cerbuna 12, 50009 Zaragoza, Spain

${ }^{f}$ Instituto de Instrumentación para Imagen Molecular (I3M), Universitat Politècnica de València, Camino de Vera, s/n, Edificio $8 B, 46022$ Valencia, Spain

${ }^{g}$ Joint Institute for Nuclear Research (JINR), Joliot-Curie 6, 141980 Dubna, Russia

${ }^{h}$ Centro de Investigaciones, Universidad Antonio Nariño, Carretera 3 Este No. 47A-15, Bogotá, Colombia

${ }^{i}$ Department of Physics and Astronomy, Iowa State University, 12 Physics Hall, Ames, Iowa 50011-3160, USA

${ }^{j}$ Instituto Gallego de Física de Altas Energías (IGFAE), Univ. de Santiago de Compostela, Campus sur, Rúa Xosé María Suárez Núñez, s/n, 15782 Santiago de Compostela, Spain

${ }^{k}$ Departamento de Física Teórica, Universidad Autónoma de Madrid, Campus de Cantoblanco, 28049 Madrid, Spain

${ }^{l}$ Institute of Nanostructures, Nanomodelling and Nanofabrication (i3N), Universidade de Aveiro, Campus de Santiago, $3810-193$ Aveiro, Portugal ${ }^{m}$ Instituto de Física Teórica (IFT), UAM/CSIC, Campus de Cantoblanco, 28049 Madrid, Spain

${ }^{n}$ Dpto. de Mecánica de Medios Continuos y Teoría de Estructuras, Univ. Politècnica de València, Camino de Vera, s/n, 46071 Valencia, Spain ${ }^{o}$ Escola Politècnica Superior, Universitat de Girona, Av. Montilivi, s/n, 17071 Girona, Spain

${ }^{p}$ Department of Physics and Astronomy, Texas AE M University, College Station, Texas 77843-4242, USA

\begin{abstract}
Ionization and scintillation produced by nuclear recoils in gaseous xenon at approximately 14 bar have been simultaneously observed in an electroluminescent time projection chamber. Neutrons from radioisotope $\alpha$-Be neutron sources were used to induce xenon nuclear recoils, and the observed recoil spectra were compared to a detailed Monte Carlo employing estimated ionization and scintillation yields for nuclear recoils. The ability to discriminate between electronic and nuclear recoils using the ratio of ionization to primary scintillation is demonstrated. These results encourage further investigation on the use of xenon in the gas phase as a detector medium in dark matter direct detection experiments.
\end{abstract}

Keywords: dark matter, high pressure xenon gas, WIMP, neutrinoless double beta decay, nuclear recoils PACS: 29.40.Mc, 61.25.Bi, 95.35.+d

*Corresponding author. Present address: Instituto de Física Corpuscular (IFIC), CSIC \& Universitat de València, Calle Catedrático José Beltrán, 2, 46980 Paterna, Valencia, Spain. Email address: jrenner@lbl.gov

${ }^{1}$ Spokesperson

${ }^{2}$ Deceased

Preprint submitted to Nuclear Instruments and Methods A

\section{Introduction}

Xenon has been the detection medium of choice in multiple experiments searching for rare physics events due to its favorable properties as a detection medium $[1,2]$ including the availability of two channels of en- 
ergy measurement, scintillation and ionization, that can be accessed simultaneously in a single detector. In particular, recent experiments have employed liquid xenon in searching for interactions of WIMP (Weakly Interacting Massive Particle) dark matter [3, 4, 5], and neutrinoless double-beta $(0 v \beta \beta)$ decay [6]. Both of these processes have strong implications in fundamental physics. WIMPs are strong candidates to be a possible constituent of cold dark matter (see for example [7]), thought to make up the majority of matter in the universe. The observation of $0 v \beta \beta$ decay (see for example [8]) would establish the Majorana nature of the neutrino and provide information on the absolute value of the neutrino masses and the neutrino mass hierarchy.

WIMPs interact via the electroweak force, allowing them to elastically scatter off nuclei, and so the signature of a WIMP in a pure xenon detector would be the recoil of a xenon nucleus, in which the energetic nucleus excites and ionizes xenon atoms to produce primary scintillation photons and electron-ion pairs. $\mathrm{Nu}-$ clear recoils have been observed and well-characterized in liquid xenon. In particular, it is known that the scintillation and ionization yields of nuclear recoils are lower, or quenched, relative to those of energetic electrons (electronic recoils) of the same kinetic energy. A model that predicts these yields based on the existing measurements has been constructed in [9]. Experiments in liquid xenon have also clearly shown that the amount of quenching in both scintillation and ionization is not the same, enabling one to discriminate between electronic recoils and the nuclear recoil signals of interest to dark matter detection by using the ratio of ionization to primary scintillation (see for example $[10,11,12]$ ).

The use of xenon in the gas phase may provide several advantages that would imply greater sensitivity in searches for dark matter and $0 v \beta \beta$ decay. In particular, the gas phase offers improved energy resolution [13] over the liquid phase, largely due to the observed significant fluctuation in energy deposition between the ionization and scintillation channels [14] in liquid Xe. Though this can be corrected by combining both channels to recover some of the lost energy resolution, as done in [6], the inability to achieve light collection efficiencies beyond $\sim 20 \%{ }^{3}$ limits overall resolution in the combined signal due to Poisson fluctuations inherent to the detection of a relatively small amount of primary scintillation. In the gas phase, good energy resolution is realizable using only the $\mathrm{S} 2$ signal. Better energy resolution could lead to improved electron/nuclear recoil

${ }^{3}$ The LUX experiment (see Ref. [3]) achieved an average detection efficiency of $14 \%$ for primary scintillation. discrimination. Under the right conditions and possibly with the addition of a molecular additive to the pure xenon gas, the amount of electron-ion recombination in nuclear recoil tracks may show a dependency on the orientation of the drift electric field relative to the orientation of the track, thereby providing information about the direction of the incident WIMP $[15,16]$.

Here we report data on the ionization and scintillation of nuclear recoils in gaseous xenon. Further details of this study can be found in [17] and [18]. In addition, scintillation and ionization of nuclear recoils were previously presented in [19]. The experiment was performed with a high pressure xenon gas time projection chamber (TPC) constructed as a prototype for NEXT (Neutrino Experiment with a Xenon TPC), called the NEXT prototype for research and development towards detection of neutrinoless Double Beta and Dark Matter (NEXT-DBDM). NEXT will search for $0 v \beta \beta$ decay with an electroluminescent TPC containing $100 \mathrm{~kg}$ of enriched $\left(91 \%{ }^{136} \mathrm{Xe}\right)$ xenon. Should potential advantages be found in using gaseous xenon to search for dark matter, one could comtemplate a simultaneous $0 v \beta \beta$ and dark matter search with a ton-scale gaseous xenon detector. A clear understanding of nuclear recoils in gaseous xenon is a critical first step in this direction.

\section{Experimental Setup and Calibration}

\subsection{Detector Hardware and Operation}

The NEXT-DBDM detector is described in detail in [20]. Here we summarize this description and describe the modifications made for this study. Figure 1 gives an overview of the experimental setup and source locations.

The main hardware of the TPC consists of a stainless steel cylindrical pressure vessel $(20 \mathrm{~cm}$ diameter, 33.5 $\mathrm{cm}$ length) with one end closed in an ellipsoidal shape and the other sealed via a ConFlat flange to a stainless steel lid to which the internal components forming the TPC are attached. The internal hardware consists of a hexagonal field cage separated into a drift (active) region of length $8 \mathrm{~cm}$ and an amplification region of length $5 \mathrm{~mm}$ by grids of wire mesh stretched tightly across metal frames. The active region is enclosed by PTFE panels with copper strips attached to their outer surfaces which are connected via resistors to grade the drift field. The panels are supported by thin plastic frames, and the PTFE surfaces facing the active region were coated with tetraphenyl butadiene (TPB) by dissolving the TPB in toluene and spraying it directly onto the surface using an airbrush. An array of 19, 1in. diameter Hamamatsu photomultiplier tubes (PMTs) 


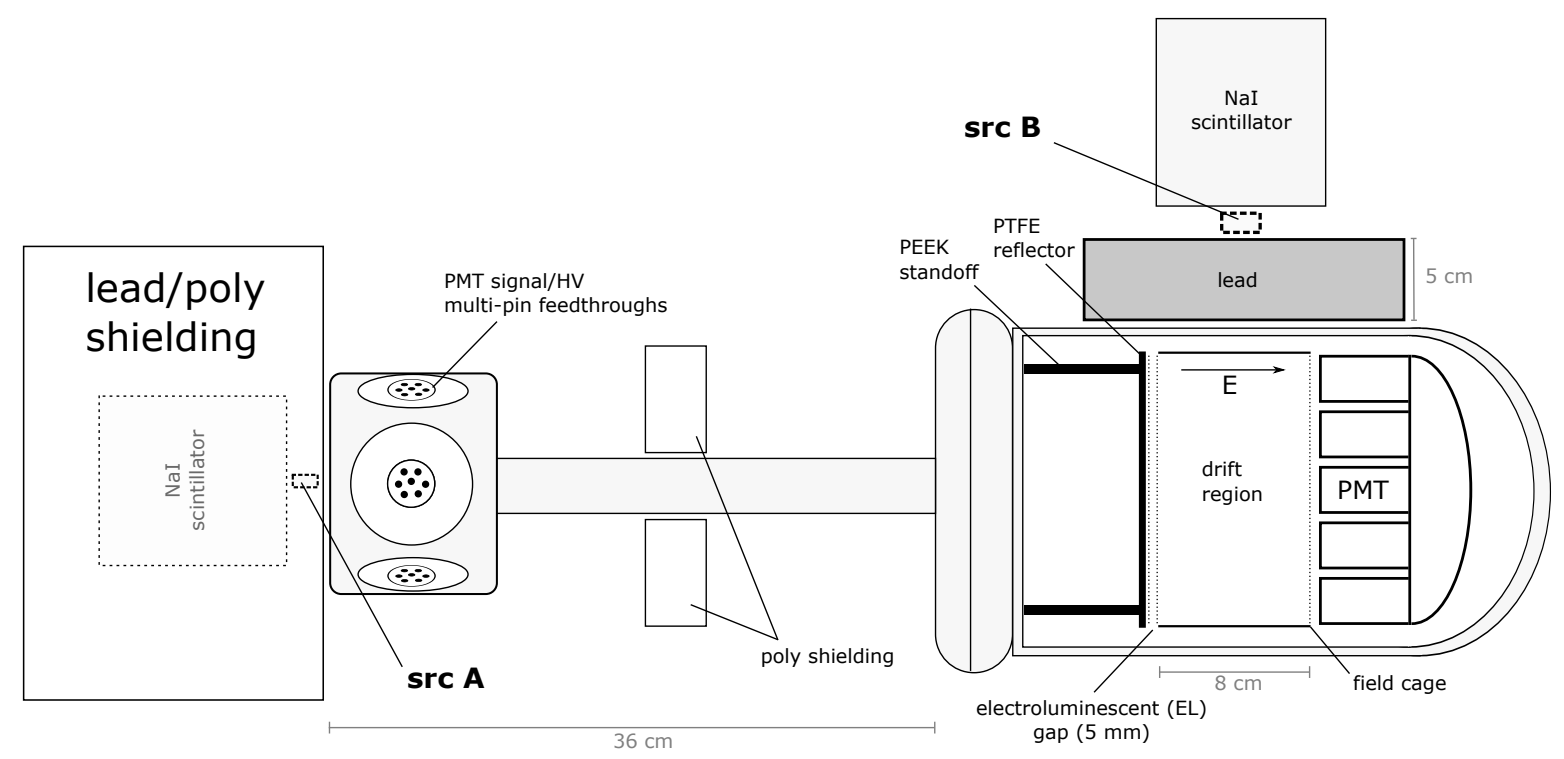

Figure 1: Schematic of experimental configuration (not drawn to scale) for datasets used in this study. When the source was at position A, both the $\mathrm{NaI}$ scintillator and the source were enclosed in the lead/poly shielding.

arranged in a hexagonal pattern is located at the end op- 135 posite to the amplification region. High voltages for the ${ }_{136}$ wire meshes are fed into the pressure vessel through the ${ }_{137}$ lid via commercial feedthroughs rated to $20 \mathrm{kV}$ at $17{ }_{138}$ bar and connected via PTFE-coated wire to the mesh 139 frames. The lid is connected by a long tube to a stain- ${ }_{140}$ less steel octagon with 8 ConFlat ports, several of which ${ }_{141}$ are occupied by multi-pin feedthroughs through which ${ }_{142}$ PMT high voltages are input to the interior of the detec- ${ }_{143}$ tor and through which the PMT signals are output. An ${ }_{144}$ opening of diameter $1.7 \mathrm{~cm}$ extends through the cen- 145 ter of the octagon and down the tube to a $2 \mathrm{~mm}$ source entrance window to the interior of the pressure vessel. ${ }_{147}$ An external sodium iodide (NaI) scintillator coupled to ${ }_{148}$ a PMT was used to tag gamma rays emitted in coinci- 149 dence with the neutrons or gamma rays of interest. The ${ }_{150}$ tagging procedure mostly served to identify events of in- ${ }_{151}$ terest but also provided some time-of-flight information (see section 3.1).

The pressure vessel is connected to a gas system allowing for full system pump-down to pressures on the order of $5 \times 10^{-5}$ Torr. The gas system also permitted ${ }_{153}$ reclamation/reintroduction of the xenon gas used in op- 154 eration to/from a steel cylinder and constant recircula- 155 tion of the xenon gas during operation through a heated 156 zirconium-based getter, to remove impurities such as ${ }^{157}$ $\mathrm{O}_{2}, \mathrm{H}_{2} \mathrm{O}$, and $\mathrm{N}_{2}$. Typically after servicing the TPC, the 158 entire system is pumped to $\sim 5 \times 10^{-5}$ torr, flushed with 159 Ar gas, pumped a second time, and filled with xenon 160 gas. The gas must be purified for several days before ${ }_{161}$ optimal electron lifetimes are realized. The system is operated at room temperature, and the temperature inside the detector was between about 22 and 26 degrees celsius depending on the location within the pressure vessel.

A typical event in the detector occurs when an energetic particle interacts in the xenon medium, producing primary scintillation in the form of $\lambda \sim 170 \mathrm{~nm}$ photons, and ionization [1]. The primary scintillation is detected immediately by the PMTs and constitutes the signal denoted as S1. The ionization is drifted via an electric field to the amplification region, a narrow region of high field in which the electrons are accelerated to energies sufficient to repeatedly excite but not ionize the xenon atoms in the medium. Each excitation results in the emission of a xenon UV photon so that each individual electron traversing the high field region produces a number of photons $g$ equal to, at room temperature $[21,22]$

$$
g=140(E / p-0.83) p \Delta x,
$$

where $\Delta x$ is the thickness in $\mathrm{cm}$ of the region of high field $E$, given in $\mathrm{kV} / \mathrm{cm}$, and $p$ is the gas pressure in bar. This process, called electroluminescence, provides a means of amplification with lower fluctuations than amplification based on charge multiplication and results in a light signal denoted as S2 that is proportional to the number of ionization electrons produced in the event.

The TPB on the walls surrounding the active region shifts a significant fraction of the xenon scintillation 
light to the visible regime $(\lambda \sim 430 \mathrm{~nm})$ at which the ${ }_{213}$ quantum efficiency of the PMTs is higher. The presence 214 of the TPB provided about a factor of 2 increase in light 215 collection efficiency and was essential to observing the 216 low S1 signals produced by nuclear recoils discussed 217 in Sec. 3. This can be attributed to the combination 218 of several factors including a high UV to visible con- 219 version efficiency of about $80 \%$ at $170 \mathrm{~nm} \mathrm{[23],} \mathrm{the} \mathrm{in-} 220$ creased reflectivity of PTFE at wavelengths of $\sim 400 \mathrm{~nm} 221$ (>95\% at normal incidence) over wavelengths of $\sim 170 \quad 222$ $\mathrm{nm}$ (about $60 \%$ at normal incidence) [24], and increased 223 PMT quantum efficiency at higher wavelengths.

All PMT signals are sampled continuously at a rate of 225 $100 \mathrm{MHz}$ using a Struck SIS3302 digitizer and buffered 226 in waveforms of 16384 samples that are stored in dig- 227 itizer memory when a trigger constructed from a net- 228 work of NIM modules is activated. The events are read 229 out to a desktop computer in blocks of 512 and pro- 230 cessed using an automated data management and anal- 231 ysis system based on ROOT [25] and FMWK [26]. In 232 the analysis, the signals from the 19 PMTs are base- $2 з 3$ line subtracted and summed, and peaks corresponding 234 to PMT pulses are located and integrated to give a num- 235 ber of detected photons (see Fig. 4 for an example wave- 236 form). Each peak is considered as a candidate S1 or S2 ${ }_{237}$ pulse based on its width and, when the arrival time of $\mathrm{S} 1$ in the waveform was fixed due to tagging with an 238 external scintillator, its arrival time. Once a single S1 239 pulse and one or more S2 pulses have been identified, 240 the drift time of the ionization produced in the event 241 can be determined as the difference in time between the 242 beginning of the $\mathrm{S} 1$ pulse and the centroid in time of ${ }_{243}$ the S2 pulse. One or more S2 peaks are possible in a 244 single event if the ionization track has multiple com- 245 ponents that arrive at the amplification region at distinct 246 times. This could occur, for example, if a xenon fluores- 247 cent X-ray were produced and traveled several $\mathrm{cm}$ before 248 making a distinct ionization track of its own. Key quan- 249 tities from each event such as the integrated numbers 250 of photons in the S1 and S2 signals, the drift time, and 251 pulse integration and timing information from the exter- 252 nal NaI scintillator are stored in a ROOT data structure ${ }_{253}$ to facilitate access.

\subsection{Sources and Configuration}

The data discussed in this study were acquired using radiation emitted from four different radioactive sources - two producing only gamma radiation and two radioiso- 258 tope neutron sources producing energetic $(\sim 1-10 \mathrm{MeV}) \quad 259$ neutrons. The neutron-producing ${ }^{9} \operatorname{Be}(\alpha, \mathrm{n}){ }^{12} \mathrm{C}$ reaction 260 that occurs within the sources often leads to the emis- 261 sion of a high-energy gamma ray in coincidence with 262 the emitted neutron. This gamma ray has an energy of either $4.4 \mathrm{MeV}$ or $7.7 \mathrm{MeV}$. To more efficiently isolate neutron-induced events in this study, the detection of a $4.4 \mathrm{MeV}$ gamma ray using a $\mathrm{NaI}$ scintillator was included as one of the trigger conditions when data was taken with a neutron source. Further details on the neutron production mechanisms and spectra of the radioisotope neutron sources are discussed in detail in the Appendix.

The configurations of the sources used in this study are itemized in the following subsections. Of the 19 PMTs in the energy plane, 18 were operational during the acquisition of all datasets, and all datasets were taken with a drift field of $370 \mathrm{~V} / \mathrm{cm}$ at a gas pressure of approximately 14 bar. These values of drift field and pressure were chosen based on experimental and operational constraints. In particular, the drift field could not be increased further without causing excessive sparking at one of the high voltage feedthroughs unless the electroluminescent (EL) gain was reduced, and so it was chosen as a compromise between EL gain and drift field strength. The good energy resolution offered by the gas phase [13] is expected to be obtainable at all pressures at which the detector was capable of operation (up to 17 bar).

\subsection{1. ${ }^{241} \mathrm{Am} / \mathrm{Be}$ Neutron Source}

An americium-beryllium neutron source containing a mass of ${ }^{241} \mathrm{Am}$ with approximately $56 \mathrm{mCi}$ of activity was positioned in source location A as shown in Fig. 1, just in front of the long tube connecting to the pressure vessel lid. The source was surrounded by a layer of lead about $2 \mathrm{in}$. thick and a layer of polyethylene also about 2 in. thick, such that it was only exposed in the direction along the tube. Additional polyethylene shielding was placed along the tube to collimate the incident neutron flux to the cross-sectional area of the tube. The $\mathrm{NaI}$ scintillator was placed within the shielding nearby the source to tag $4.4 \mathrm{MeV}$ gamma rays emitted from it. In this configuration, neutrons were emitted far enough from the xenon volume that their interactions could be distinguished from those induced by gamma rays. This could be done by using the time-of-flight measured as the time difference in the arrival of the $\mathrm{S} 1$ produced in the TPC and the gamma ray tagged in the NaI scintillator.

\subsection{2. ${ }^{238} \mathrm{Pu} / \mathrm{Be}$ Neutron Source}

A plutonium-beryllium neutron source containing a mass of ${ }^{238} \mathrm{Pu}$ with approximately $10 \mathrm{mCi}$ of activity was positioned at the side of the pressure vessel between the $\mathrm{NaI}$ scintillator and a lead brick at source location $\mathrm{B}$ 
of Fig. 1. The presence of the lead greatly reduced the background due to gamma rays emitted as products of the neutron-generating $(\alpha, \mathrm{n})$ reaction.

\subsection{3. ${ }^{22} \mathrm{Na}$ Gamma Source}

A sodium source containing a mass of ${ }^{22} \mathrm{Na}$ with approximately $10 \mu \mathrm{Ci}$ of activity was placed at the side of the pressure vessel, similar to the configuration shown for source B in Fig. 1, though no lead block was present. The source was positioned about $16 \mathrm{~cm}$ from the side of the pressure vessel, and the NaI scintillator was positioned several centimeters away from the source to avoid pileup. Two collinear $511 \mathrm{keV}$ gamma rays are emitted back-to-back from the source. One gamma ray was tagged with the NaI scintillator while the other was incident on the xenon volume.

\subsection{4. ${ }^{137}$ Cs Gamma Source}

A cesium source containing a mass of ${ }^{137} \mathrm{Cs}$ with approximately $1 \mathrm{mCi}$ of activity was contained in a lead enclosure to which a cylindrical lead collimator with an opening of diameter $3.5 \mathrm{~mm}$ was fitted at one end. The resulting collimated beam of $662 \mathrm{keV}$ gamma rays was placed at source location A as shown in Fig. 1 and pointed down the tube connecting the octagon and pressure vessel through the $2 \mathrm{~mm}$ thick source entrance window.

\subsection{Detector Calibration with a ${ }^{137}$ Cs Source}

Before discussing the characterization of nuclear recoils, we establish a gamma-based calibration of the detector using a ${ }^{137} \mathrm{Cs}$ source (configuration 4 of Sec. 2.2). This also serves to demonstrate the ability of the instrumentation to process the many photons detected in a high-energy gamma event without signal saturation while, under the same operating conditions, retaining the ability to process the few $\mathrm{S} 1$ photons detected in a nuclear recoil event. A wide photon detection range would be a necessary component of a simultane- ${ }_{328}$ ous search for neutrinoless double-beta decay and dark ${ }_{329}$ matter.

Gamma rays of energy $662 \mathrm{keV}$ were directed axially ${ }_{331}$ through the center of the TPC lid, and the S1 and S2 ${ }_{332}$ signals were examined to determine key xenon properties such as the amount of energy required to produce a primary scintillation photon $W_{\mathrm{sc}}$, the relation used to correct for the $\mathrm{z}$-dependence (lower detection probability of photons produced farther from the PMT array) of ${ }_{334}$ S1, and the light collection efficiency $\epsilon$ at the EL plane. 335 We first assume a value for the amount of energy re- 336 quired to produce an ionization electron $W_{\mathrm{i}}=24.7 \pm 1.1$ eV (see Sec. 3.2 of [21]).

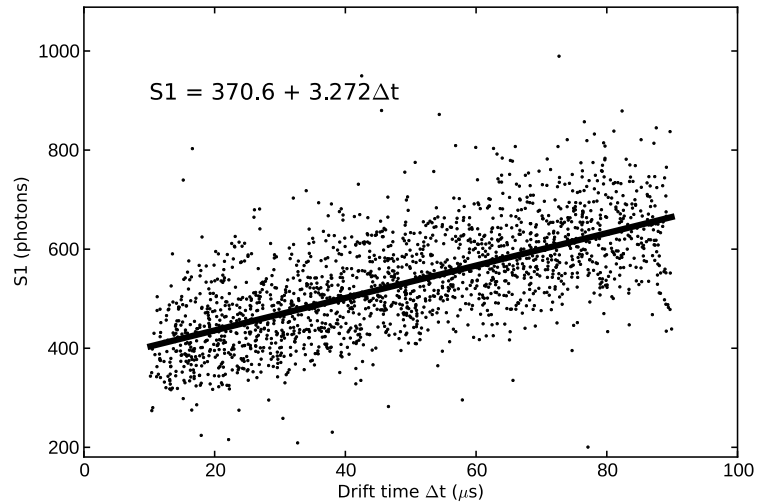

Figure 2: $\mathrm{S} 1$ vs. drift time (effectively the $\mathrm{z}$-coordinate) for events determined to fall in the $662 \mathrm{keV}$ peak in a ${ }^{137} \mathrm{Cs}$ calibration run. The linear fit was used to determine a correction factor to compute the S1 value for each event as if it occurred at the EL plane $(z=0)$.

Events corresponding to full-energy depositions of $662 \mathrm{keV}$ gamma rays were isolated as a peak in the S2 distribution produced by the source. A central fiducial cut was made according to the weighted average (x, y) location of the event determined using the distribution of electroluminescent light produced on the PMT plane, and the events were corrected for electron attachment by multiplying by a z-dependent exponential factor corresponding to an electron lifetime of $\tau_{e} \approx 8.3 \mathrm{~ms}$. Events with drift times less than $10 \mu s$ or greater than $90 \mu s$ were also cut to ensure that the events considered were produced within the drift region and with no overlap between S1 and S2. The ${ }^{137} \mathrm{Cs} \mathrm{S} 2$ photopeak was found to lie at $S_{2}=561617 \pm 92$ photons. Operating at EL gain $g=734 \pm 89$ photons $/ \mathrm{e}^{-}$, we calculate for the light collection efficiency at the EL plane,

$$
\epsilon=\frac{S_{2} \cdot W_{\mathrm{i}}}{g \cdot E_{\gamma}}=0.0285 \pm 0.0037,
$$

where $E_{\gamma}=661657 \pm 3 \mathrm{eV}$ [27] is the energy of the gamma ray. The geometrical z-dependence of S1 detection efficiency was determined by plotting the integrated S1 values of the events in the ${ }^{137} \mathrm{Cs}$ S2 photopeak vs. event drift time (see Fig. 2). A linear dependence is observed,

$$
S_{1}=S_{1,0}+k \Delta t
$$

where $\Delta t$ is the drift time and $S_{1,0}$ is a constant corresponding to the number of $\mathrm{S} 1$ photons detected at the EL plane for the $662 \mathrm{keV}$ energy deposition. It was determined that $S_{1,0}=370.6 \pm 4.0$ photons and $k=3.272 \pm 0.076$ photons $/ \mu$ s, so that the $\mathrm{S} 1$ signal de- 
tected for an event with drift time $\Delta t$, denoted as $S_{1}(\Delta t)$, could be corrected for its z-dependence as

$$
S_{1}^{\prime} \equiv S_{1,0} \cdot \frac{S_{1}(\Delta t)}{S_{1,0}+k \Delta t}=\frac{S_{1}(\Delta t)}{1+\left(k / S_{1,0}\right) \Delta t} .
$$

Knowing how to correct the S1 signal to its value at the EL plane, and knowing the light collection efficiency at the EL plane, we can compute the energy required to produce an S1 photon $W_{\text {sc }}=\left(E_{\gamma} / S_{1}\right) \epsilon^{\prime}$, where $\epsilon^{\prime}=\epsilon / \Omega$ and $\Omega$ is a factor accounting for the optical effect of the EL wire mesh grids. This correction is necessary because $\epsilon$ is calculated using S2 light produced in the EL gap between two grids while the $\mathrm{S} 1$ light is produced in the active region. The factor $\Omega$ was determined by a Monte Carlo simulation in which photons were generated within the EL gap and just in front of the EL gap, and the resulting efficiencies in the two cases were compared. The grids in the simulation were given the nominal transparency of the physical mesh grids, equal to $88 \%$ at $0^{\circ}$ incidence angle, and from the Monte Carlo, the relative light collection efficiency was determined to be described by $\Omega=0.83 \pm 0.08$, assuming $10 \%$ errors. Using Eq. 2, we find

$$
W_{\mathrm{sc}}=\frac{S_{2} \cdot W_{\mathrm{i}}}{S_{1} \cdot g \cdot \Omega}=61.4 \pm 18.0 \mathrm{eV},
$$

with an applied drift field of $370 \mathrm{~V} / \mathrm{cm}$, and where we ${ }^{386}$ have assumed an additional systematic error of $15 \mathrm{eV}{ }^{387}$ obtained from Monte Carlo studies (see Sec. 3.3). 388

The obtained $W_{\text {sc }}$ is lower than those obtained in 389 other references including $W_{\mathrm{sc}}=76 \pm 12 \mathrm{eV}$ [28] and 390 $W_{\mathrm{sc}}=111 \pm 16 \mathrm{eV}$ [29], though these experiments were ${ }^{391}$ carried out under significantly different operating con- 392 ditions and in one case [28] in a mixture of $90 \% \mathrm{Xe} /{ }^{393}$ $10 \%$ He gas. Note that in studies of liquid xenon detec- 394 tors [9], strong dependencies of $W_{\mathrm{sc}}$ on recombination have lead to the definition of an average $W$ in terms of the number of xenon excitations $N_{\mathrm{ex}}$ and ionizations $N_{\mathrm{i}}$ produced during the creation of the track, such that the 396 total energy deposited $E_{\gamma}=W\left(N_{\mathrm{ex}}+N_{\mathrm{i}}\right)$. For this setup 397 assuming $E_{\gamma}=W_{i} N_{i}$ with $W_{\mathrm{i}}=24.7 \mathrm{eV}$, we would have 398 $W=W_{\mathrm{i}} /\left(1+N_{\mathrm{ex}} / N_{\mathrm{i}}\right)=17.6 \mathrm{eV}$ using $N_{\mathrm{ex}} / N_{\mathrm{i}}=0.4$ for 399 noble gases from [30].

We also note that both S1 and S2 pulses appeared to 401 possess long tails, a property which was not present dur- 402 ing previous operation before TPB was placed on the 403 walls of the field cage. While the S2 light is produced 404 over a timespan of several microseconds to tens of mi- 405 croseconds, the S1 light arrives over a short timescale, 406 allowing for the characterization of the tail accompa- 407 nying a single, fast pulse of light. The S1 pulses were 408

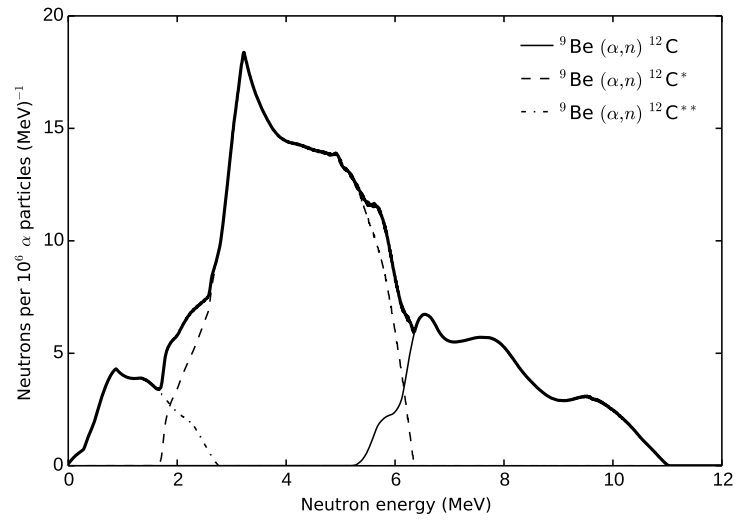

Figure 3: Calculated neutron emission spectrum for a ${ }^{241} \mathrm{Am} / \mathrm{Be}$ neutron source considering only the neutrons produced in the ${ }^{9} \mathrm{Be}(\alpha, \mathrm{n}){ }^{12} \mathrm{C}$ reaction. The spectrum is divided into three components for which the resulting carbon nucleus is found in the ground (solid line), first excited (dashed line), or second excited (dot-dashed line) state. The bold solid line shows the sum of all three components. The neutrons of greatest interest to this study are those for which the carbon nucleus is left in the first excited state and decays emitting a 4.4 $\mathrm{MeV}$ gamma ray, as these neutrons create nuclear recoils of energies that can be detected by the present setup and their emission can be tagged by detecting the coincident gamma ray with an external scintillator.

found to be well described by a two component exponential, one with a short decay time constant of $\tau_{s} \approx$ $100 \mathrm{~ns}$, and one with a longer decay time constant of $\tau_{l} \approx 1.4 \mu \mathrm{s}$. The $100 \mathrm{~ns}$ component has been observed previously (see for example [31]) as the de-excitation of the triplet exciton state in gaseous xenon, but the longer component is likely to be linked to the introduction of the TPB to the interior of the detector. Since TPB itself is not expected to produce such an effect, it may be the result of an agent present in the toluene-based solution employed in the coating process.

\section{Nuclear Recoils in High Pressure Xenon Gas}

\subsection{Analysis of Experimental Data}

In this section, we present data taken with the radioisotope neutron sources, and we first show the expected spectrum of emitted neutrons from such sources. The neutron spectrum from a ${ }^{241} \mathrm{Am} / \mathrm{Be}$ source is shown in Fig. 3 and was calculated assuming the source consisted of a volume filled with beryllium throughout which the isotope ${ }^{241} \mathrm{Am}$ was uniformly distributed. Further details and a description of the relevant calculations can be found in the Appendix.

The neutrons emitted from the radioisotope sources used may be accompanied by gamma rays, and often the neutrons themselves scatter inelastically off nuclei 


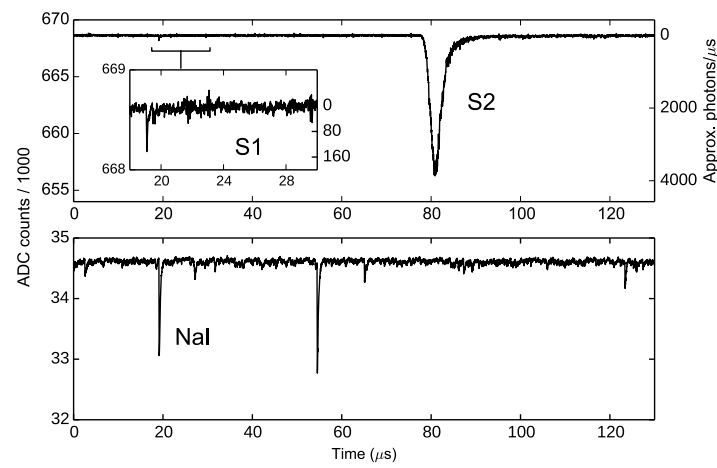

Figure 4: A candidate neutron event. The (unweighted) sum of all 4 PMT signals is shown above, and the NaI scintillator waveform acquired simultaneously is shown below. Note the $\mathrm{S} 1$ in coincidence with a pulse in the PMT coupled to the NaI scintillator, followed by the single, Gaussian-like S2 pulse indicative of a pointlike energy dis- 449 tribution in the TPC. The slight bulge in the right tail of the S2 pulse is due to extended light emission from the presence of the TPB coating on the PTFE walls of the field cage. The right-hand scale of the PMT sum waveform shows the approximate number of photons per microsecond corresponding to a given location on the y-axis assuming equal PMT weighting factors. in the environment (in the xenon or surrounding de- 457 tector hardware), resulting in the emission of various 458 gamma rays in the de-excitation of the nuclei. There- 459 fore, careful analysis is necessary to isolate the nuclear recoil events, and to do so, we make a sequence of analysis cuts on the data to remove gamma-induced events and finally identify a band of events at low values of S1 460 and $\mathrm{S} 2$ that consists primarily of nuclear recoils.

The data presented here, consisting of 3682304 events in total, was acquired over about 577 hours, during which time the acquisition system was actively recording events at a rate of about $3 \mathrm{~Hz}$ for about 346 hours. The rate of acquisition was stable throughout the majority of the run, and 6 blocks of 512 events were removed due to anomalous rates. Most of the downtime was due to the readout and storage of events from the digitizer. Events occurring within 5 minutes of occasional high voltage breakdown within the detector were discarded. This eliminated about $3.8 \%$ of the events, thus slightly lowering the effective live-time.

The analysis cuts were performed in a sequence consisting of: tagging and single-pulse event identification, time-of-flight, diffusion, and radial cuts.

Tagging and single-pulse identification cuts. A typ- 478 ical tagged neutron candidate event is shown in Fig. 4. 479 The S1 signal and NaI pulses were fit to a function of 480 the form

$$
f(x)=p_{0}+\frac{p_{1} e^{-\left(x-p_{2}\right) / p_{4}}}{1+e^{-\left(x-p_{2}\right) / p_{3}}},
$$

event is determined for properly tagged events as the difference between the arrival times of the $\mathrm{S} 1$ and $\mathrm{NaI}$ pulses and is given by

$$
\tau=\left[\frac{1+E_{0} / K}{\sqrt{1+2 E_{0} / K}}\right](d / c)+\Delta t_{\mathrm{off}}
$$

where $E_{0}$ and $K$ are the rest and kinetic energies of the particle, $d \approx 50 \mathrm{~cm}$ is the distance traveled by the particle from the source to the point of detection, $c$ is the speed of light, and $\Delta t_{\text {off }}$ is an offset time due to the trigger and electronics. The tagging procedure serves to eliminate a significant amount of gamma background, and a clear peak in time-of-flight due to events induced by gammas from the source is not evident in the overall time-of-flight distribution. However, by examining only higher energy depositions consisting of a relatively higher fraction of gamma-induced events, two peaks are apparent in the time-of-flight distribution.

Figure 5 shows the relevant regime of the time-offlight distribution for high-S2 events, in which the leftmost peak corresponds to events produced by gammas emitted by the source. The fit shown to a sum of two Gaussians gives for the component corresponding to the left peak, a mean of $\mu_{\gamma} \equiv \mu_{1}=-6.24$ and sigma $\sigma_{\gamma} \equiv$ $\sigma_{1}=0.429$. We can then solve using Eq. 7 with $E_{0}=0$, $c \approx 30 \mathrm{~cm} / \mathrm{ns}$ and $\tau=\mu_{\gamma}$ for $\Delta t_{\text {off }}=\mu_{\gamma}-d / c \approx-64$ ns. Since for an active region of length $8 \mathrm{~cm}$ all gammas emitted from the source should arrive within a time 


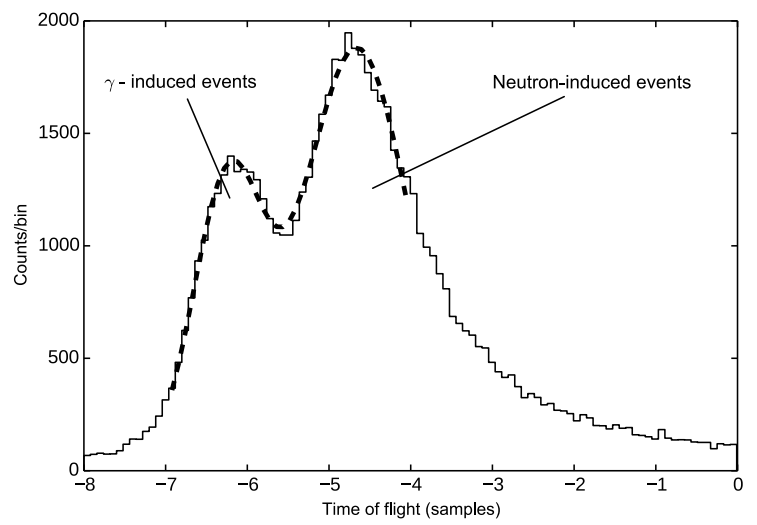

Figure 5: Time-of-flight distribution $(1 \mathrm{sample}=10 \mathrm{~ns})$ for properly tagged events with $\mathrm{S} 2>175000$ photons and 30 photons $<\mathrm{S} 1<500$ photons (corrected for $\mathrm{z}$-dependence). The left peak in the distribution is due to gammas originating from the source, and the right peak is due to events induced by neutrons from the source. The fit to a sum of two Gaussians is shown for which $\mu_{1}=-6.24, \sigma_{1}=0.429$ (left) and $\mu_{2}=-4.66, \sigma_{2}=0.647$ (right). interval of $8 \mathrm{~cm} / c=0.27 \mathrm{~ns}$, which is significantly less ${ }^{512}$ than $\sigma_{\gamma}=4.29 \mathrm{~ns}$, we can use $\sigma_{\gamma}$ as a measure of the ${ }^{513}$ time-of-flight resolution, noting that for nuclear recoil ${ }^{514}$ events we expect the resolution to be poorer due to their ${ }^{515}$ lower S1 signals. These S1 signals may consist of only ${ }^{516}$ several photons arriving within a time interval relatively ${ }^{517}$ large compared to the duration of a single photoelectron ${ }^{518}$ signal in a PMT, thereby producing inconsistencies in ${ }^{519}$ the fit to Eq. 6.

Figure 6 shows the time-of-flight distribution for lowenergy S2 events. Here the time-of-flight has been cal- ${ }^{522}$ culated in nanoseconds and the offset $\Delta t_{\text {off }}$ removed. ${ }^{523}$ Cuts were made according to the maximum and min- ${ }^{524}$ imum times-of-flight generated in a Monte Carlo us- ${ }^{525}$ ing the calculated neutron source spectrum described in ${ }^{526}$ Sec. 3.1, allowing for an additional $8 \mathrm{~ns}$ (approximately ${ }^{527}$ $2 \sigma_{\gamma}$ ) on both ends of the cut range. Any event with ${ }^{528}$ time-of-flight within the selected region shown in Fig. 6529 was considered to have passed the time-of-flight cuts. $\quad{ }_{530}$

Diffusion cuts. Electron diffusion provides an addi- ${ }^{531}$ tional means of confirming the validity of an event with 532 a single $\mathrm{S} 2$ pulse. If the selected $\mathrm{S} 1$ truly corresponds to ${ }_{533}$ the primary scintillation produced by the ionization col- ${ }_{534}$ lected as S2, the width of the S2 pulse will increase with 535 the drift time of the ionization approximately as [32] 536

$$
\sigma^{2}=\sigma_{0}^{2}+10 D_{L}^{2} \bar{t} / v_{d},
$$

where $\sigma_{0}^{2}$ is a constant determined by the drift time of ${ }_{540}$ the electron across the electroluminescent (EL) gap, $D_{L}{ }_{541}$ is the longitudinal diffusion constant in $\mathrm{mm} / \sqrt{\mathrm{cm}}, v_{d}{ }_{542}$

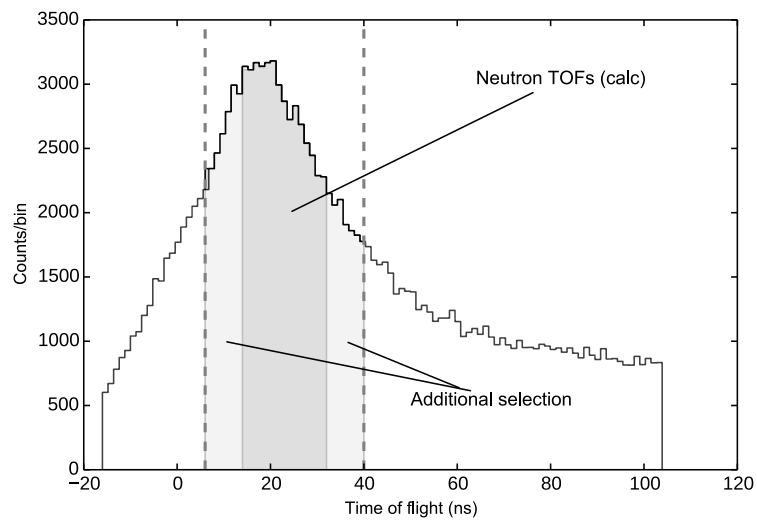

Figure 6: Time-of-flight distribution for properly tagged events with S2 $<40000$ photons and S1 $<50$ photons (corrected for $\mathrm{z}$ dependence). The shaded region shows the time-of-flight cuts applied to select $6 \mathrm{~ns}<\tau<40 \mathrm{~ns}$.

is the electron drift velocity in $\mathrm{mm} / \mu \mathrm{s}$, and $\bar{t}$ is the drift time in $\mu \mathrm{s}$. Note that this relation is an approximation as it assumes a Gaussian S2 pulse, which is not strictly the case due to the extended tails attributed to the presence of the TPB and, for events originating near the EL gap, would not hold even in absence of such tails. Events for which S1 was properly selected will fall in a band described approximately by Eq. 8, shown in Fig. 7, in which the S2 pulse width was determined by a Gaussian fit. The centroid and $\pm 2 \sigma$ lines of the band were determined using a procedure similar to the one applied in [33].

Taking a maximum drift time of about $95 \mu$ s and a drift length of $8 \mathrm{~cm}$, we have $v_{d}=0.84 \mathrm{~mm} / \mu \mathrm{s}$, and using the constant and linear terms of the centroid fit line, we find $\sigma_{0}=0.89 \mu \mathrm{s}$ and $D_{L}=0.37 \mathrm{~mm} / \sqrt{\mathrm{cm}}$. A Magboltz [34] (version 9.01) calculation yields $v_{d}=$ $0.82 \pm 0.04 \mathrm{~mm} / \mu s$ and $D_{L}=0.39 \pm 0.04 \mathrm{~mm} / \sqrt{\mathrm{cm}}$. All events within the $\pm 2 \sigma$ lines are considered to have passed the diffusion cuts. A cut on drift time alone was also applied, accepting only events with drift times between 5 and $95 \mu \mathrm{s}$.

Radial cuts. Using the pattern of light cast upon the PMT plane during S2 production, an average (x,y) location for each event can be constructed. Though this method does not provide the precision of a finergranularity tracking plane, it allows for some fiducialization and, therefore, elimination of events that originate from the walls of the TPC. The average $\mathrm{x}$ and $\mathrm{y}$ coordinates are calculated by weighting the position of each PMT by the amount of S2 signal observed by that PMT. The resulting pattern is shown in Fig. 8 and has been scaled and shifted so that it is centered upon $(0,0)$ 


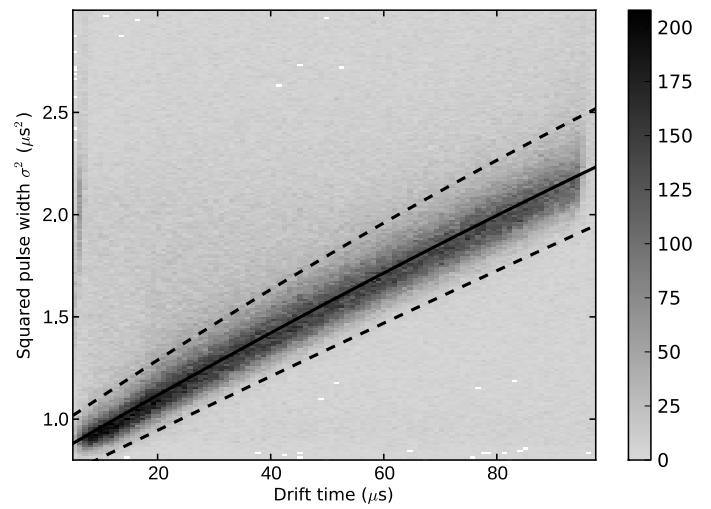

Figure 7: Pulse width $\sigma^{2}$ from a Gaussian fit to the S2 pulse in each event plotted against drift time for events near and within the diffusion band. The centroid of the band is marked with a solid line, and dashed lines define the region of \pm 2 standard deviations from the centroid. The equation of the centroid fit shown is $\sigma^{2}=0.801+0.0161 \bar{t}-$ $0.0000143 \bar{t}^{2}$. For the purposes of determining the cuts, a quadratic term has been included in the fit, but only the constant and linear terms 572 are used in the text to determine the diffusion coefficient. and its dimensions match those of the physical dimen- 575 sions of the PMT plane. This required a shift of all 576 reconstructed points by $(0.21,-0.20)$, scaling in $\mathrm{x}$ by 577 a factor of 8.71, and scaling in y by a factor of 12.6. 578 The scaling and shift procedure was necessary due to 579 the uniformity of the light pattern cast on the PMTs 580 and uncertainties in the individual PMT single-photon 581 responses. Using this procedure, a reconstruction res- 582 olution of $1-2 \mathrm{~cm}$ is anticipated based on studies done 583 in [20]. A fiducial cut of $r<3 \mathrm{~cm}$ is superimposed, 584 and events lying inside the selected region pass the cut. 585 The tight fiducial cut is made here to show more clearly 586 where the nuclear recoils lie in S1-S2 space, but this 587 cut is varied in the forthcoming discussions to obtain 588 increased statistics at the expense of more background 589 events.

From the known characteristics of nuclear recoil sig- 591 nals in liquid xenon, one suspects a class of events with 592 low S1 and S2 and a different S2/S1 slope than the elec- 593 tronic recoil events. Figure 9 shows the relevant region 594 of (S1, S2) space, including the low-energy nuclear re- 595 coil band, for all tagged events passing the single-pulse, 596 time-of-flight, diffusion, and radial cuts $(r<3 \mathrm{~cm})$ de- 597 scribed above.

\subsection{Electronic and Nuclear Recoil Discrimination}

For the purpose of dark matter searches, one is especially interested in the ability to discriminate between nuclear recoils (the potential signals) and electronic recoils (background). In liquid xenon, this can be done by

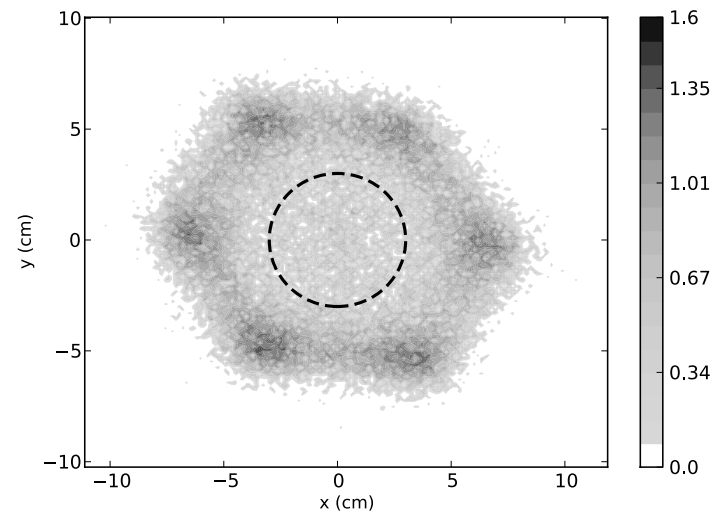

Figure 8: The distribution of average reconstructed (x,y) locations for events with $\mathrm{S} 2<40000$ photons. The shading is done on a $\log _{10}$ scale. The dashed circle defines a fiducial cut of $r<3 \mathrm{~cm}$ and encompasses $8.6 \%$ of the total number of events shown.

examining the ratio of the $\mathrm{S} 2 / \mathrm{S} 1$ signals produced in an event, as this ratio is significantly lower on average for nuclear recoils. Because the light collection efficiency in the experiments producing the present data is low (of order $3-5 \%$ ) and, therefore, the observed nuclear recoil events occupy a region of low S1 and S2 in which large fluctuations exist and the detection efficiency $\neq 1^{4}$, these data are not ideal for characterizing the full potential of the discrimination power of xenon gas. However, they can be used to demonstrate that $\mathrm{S} 2 / \mathrm{S} 1$ discrimination is possible and has potential in the gas phase. In addition, though electronic recoils can be potentially rejected using such a strategy, one is still subject to potential nuclear recoil background events produced by neutrons. These neutrons are more likely to produce a WIMP-like single-scatter event in the gas phase than in the denser liquid phase. However, if one could deduce information about the direction of a nuclear recoil in the gas phase, such information could be used to statistically separate WIMP-induced events with a preferred direction from background-induced nuclear recoil events.

Figure 10 shows the logarithm of the ratio S2/S1 plotted against $\mathrm{S} 1$ for data taken with the ${ }^{241} \mathrm{Am} / \mathrm{Be}$ neutron source and with a ${ }^{22} \mathrm{Na}$ source (configuration 3 of Sec. 2.2). The events shown passed the single-pulse, timeof-flight, diffusion, and fiducial cuts discussed in Sec. 3.1. The same number of events are shown from both datasets, which required a reduction in the number of

\footnotetext{
${ }^{4}$ Based on observations from the Monte Carlo study described in section 3.3, and not considering a constant factor determined by the $\mathrm{NaI}$ tagging efficiency, $50 \%$ detection efficiency was expected for an electronic recoil event depositing an energy of about $4.5 \mathrm{keV}$ (about 2 detected photons).
} 

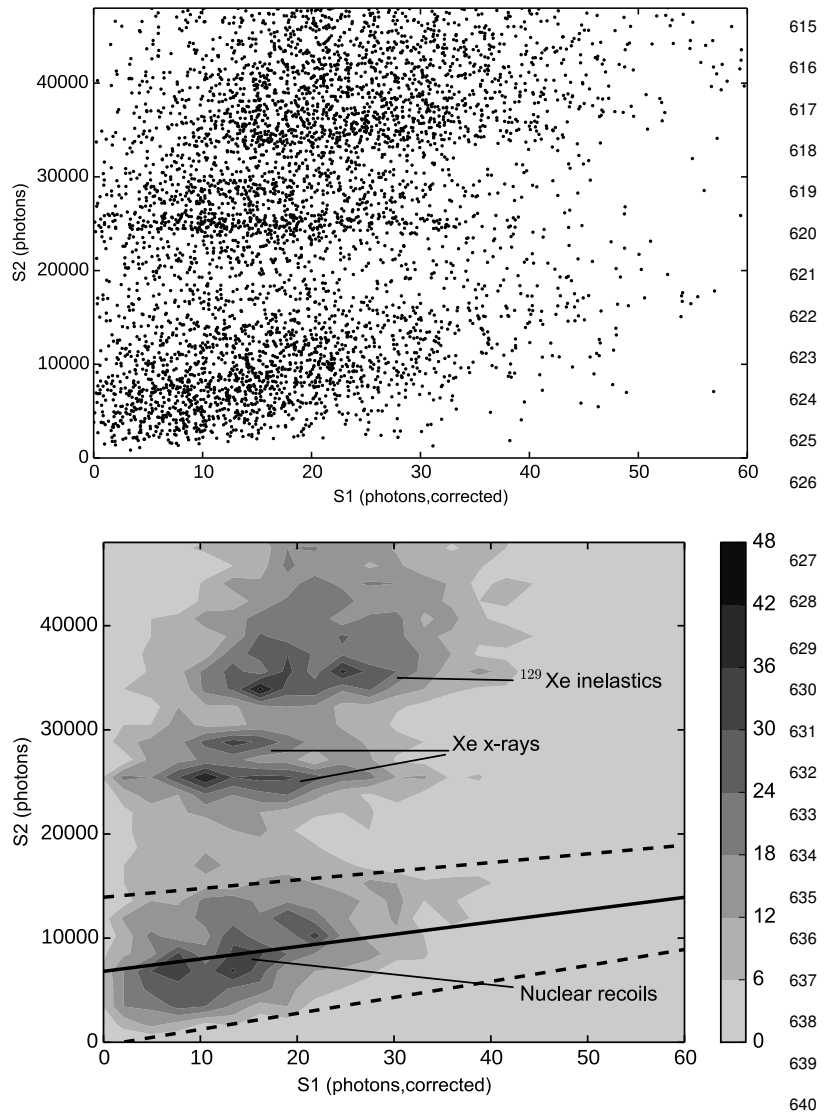

Figure 9: S1 (corrected for $\mathrm{z}$-dependence) vs. S2 signals for events passing tagging, single-pulse, time-of-flight, diffusion, and radial cuts, shown in scatter (above) and contour (below) formats. Events pro- 643 duced by neutron inelastic scattering on ${ }^{129} \mathrm{Xe}$ (approx. $40 \mathrm{keV}$ ), by 644 xenon fluorescent x-rays (approx. $29 \mathrm{keV}$ and $34 \mathrm{keV}$ ), and by nu- 645 clear recoils lie in distinct bands on the plot. The solid line shows a fit to the mean values of events distributed in 10 bins over the range S1 $\in[2,35]$ with $\mathrm{S} 2<15000$. The dotted lines are fits to the mean values 647 in these bins $\pm 2 \sigma$.

events considered from the ${ }^{241} \mathrm{Am} / \mathrm{Be}$ dataset. S1-S2 ${ }_{651}$ selection cuts were not applied so that events from both 652 electronic and nuclear recoils could be shown. The nu- 653 clear recoil events form a band clearly distinguishable 654 from the electronic recoil events, though some back- 655 ground events lie in the nuclear recoil band in the ${ }^{22} \mathrm{Na} 656$ data. $0.37 \%$ of electronic recoil events from the ${ }^{22} \mathrm{Na} 657$ data, in the S1 range over which the nuclear recoil band 658 was fit in the ${ }^{241} \mathrm{Am} / \mathrm{Be}$ data, lie below the nuclear recoil 659 band mean in figure 10. Such background events may 660 be due to several reasons, one being that at low S1, the 661 small number of photons detected are subject to more 662 significant Poisson fluctuations, resulting in poorer res- ${ }_{663}$ olution and greater likelihood of yielding an abnormally 664 large S1 for a given S2. Furthermore, it is possible to 665

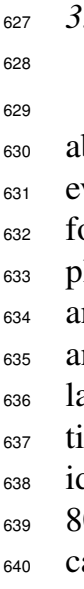

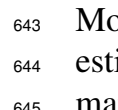

produce $\mathrm{S} 1$ in a region of the TPC from which S2 cannot be collected, for example in the small gap between the PMT plane and the wire mesh that defines the beginning of the drift region. Often a low-energy event is accompanied by additional gamma rays that escape from the active region, in which case an event with a single-pulse S2 could consist of additional gamma energy deposited but only seen in S1, yielding a range of possible S1 signals for a given S2. Such events have also been observed in liquid xenon detectors (see for example [35, 36]) and are known as multiple-scintillation single-ionization (MSSI) events.

\subsection{Monte Carlo and Estimated Ionization and Scintil- lation Yields}

The present results do not include information on the absolute energy of each nuclear recoil on an event-byevent basis, and so the ionization and scintillation yields for nuclear recoils can only be determined in principle by using the measured recoil spectrum as a whole and comparing it to expectations based on calculation and Monte Carlo simulation. For example, the peaks at larger angles in the neutron elastic scattering cross section (see Appendix) should lead to the presence of an identifiable feature in the recoil energy spectrum near $80 \mathrm{keVr}$. The presence of this feature is not statistically significant enough in the present data to make a strong definite claim, though its possible presence is investigated in [17] along with fits of experimental data to Monte Carlo spectra, and the results are used to obtain estimated nuclear recoil yields for S1 and S2. However, many uncertainties were present, including inaccuracies ergy dependence of the S1 and S2 yields, known to be non-trivial in liquid xenon [9] and for which no previously published data in gaseous xenon is known to the authors.

In this study rather than detailing a particular method of extracting information on the nuclear recoil yields, we choose values for the nuclear recoil yields (informed by the results obtained in [17]), assume a constant energy dependence, and then show that these assumptions are reasonable by comparison of experimental and Monte Carlo spectra. For this comparison we use data acquired with a ${ }^{238} \mathrm{Pu} / \mathrm{Be}$ neutron source (positioned as in experimental setup 2 described in Sec. 2.2) and a corresponding full Geant4 [37] Monte Carlo simulation. The electronic recoil yields were chosen by demanding consistency with the results obtained in Sec. 2. $W_{i}=24.7 \mathrm{eV}$ was assumed along with EL gain $g=734$, and the PMT quantum efficiency $Q$ was adjusted so that the $662 \mathrm{keV}$ S2 peak in simulation was consistent with 

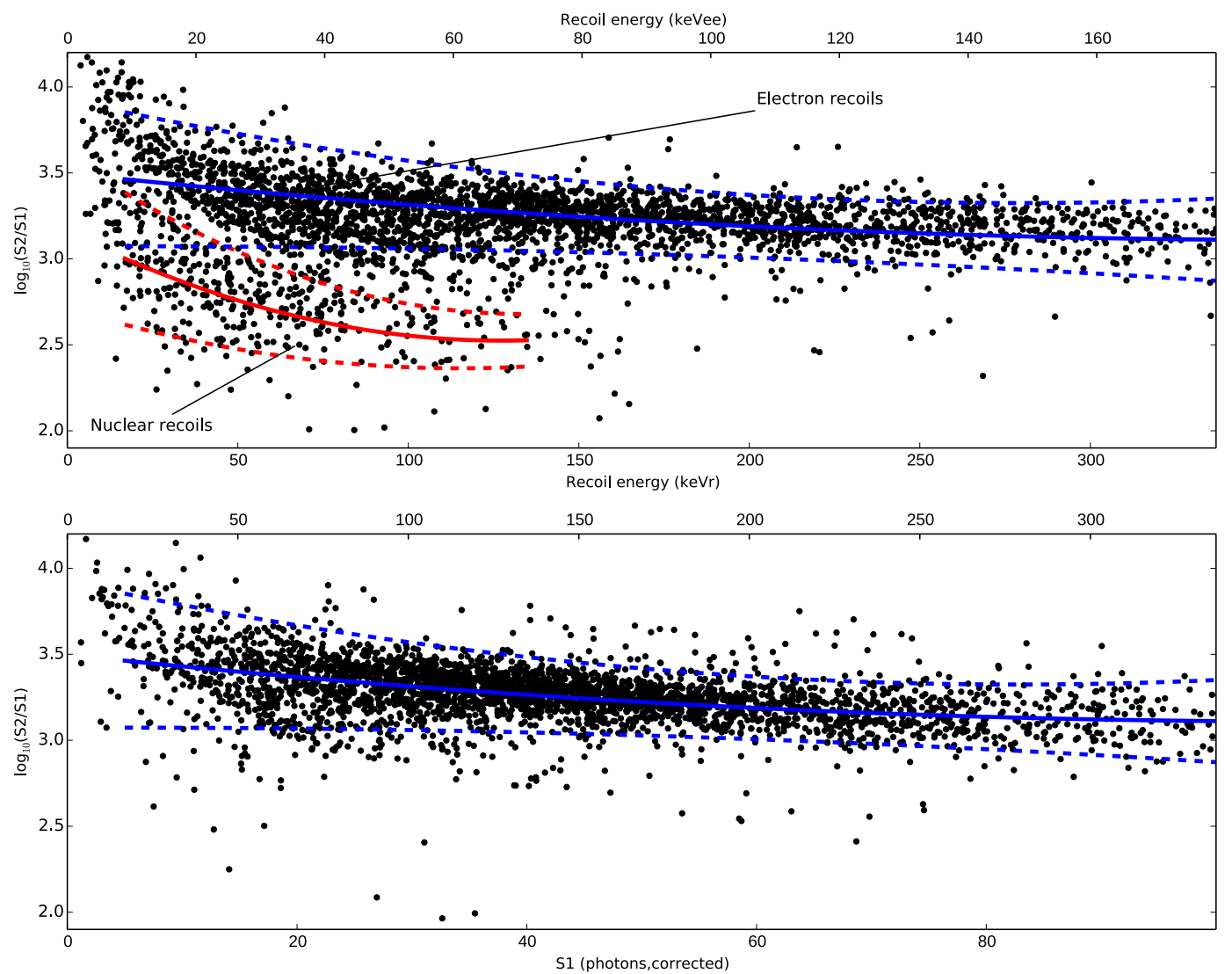

Figure 10: The logarithm of the ratio of S2/S1 signals plotted (with equivalent scales) against S1 corrected for z-dependence for data taken with the ${ }^{241} \mathrm{Am} / \mathrm{Be}$ source (above) and a ${ }^{22} \mathrm{Na}$ source (below). The $\mathrm{S} 1$ values are calibrated to their corresponding electron recoil energies (keVee) and nuclear recoil energies ( $\mathrm{keVr}$ ) using the calibration described in section 2.3 and the estimated nuclear recoil quenching factors $\alpha_{\mathrm{S} 1}$ and $\alpha_{\mathrm{S} 2}$ from section 3.3. The means and $\pm 1.5 \sigma$ lines of the electronic recoil (ER) band are shown in blue and those of the nuclear recoil (NR) band are shown in red. The ER band mean line shown on the ${ }^{241} \mathrm{Am} / \mathrm{Be}$ plot was calculated using the ${ }^{22} \mathrm{Na}$ data. The events used in calculating the NR band mean are those selected as nuclear recoils in figure 9 . The bands were calculated by fitting 2 nd-order polynomials to the mean values and mean $\pm 1.5 \sigma$ values of data points in 10 bins from $\mathrm{S} 1 \in[5,40], \mathrm{S} 2 \in[2.0,4.2]$ for the NR band and 20 bins from $\mathrm{S} 1 \in[5,100], \mathrm{S} 2 \in[3.0,4.5]$ for the ER band. About $0.37 \%$ of ER events with $\mathrm{S} 1 \in[5,40]$ (in the range considered for fitting the NR band) in the ${ }^{22} \mathrm{Na}$ data fell below the NR band mean calculated using the ${ }^{241} \mathrm{Am} / \mathrm{Be}$ data.

that found in experimental data, yielding $Q \approx 17 \%$. $W_{\text {sc }} 681$ was adjusted until the intercept $S_{1,0}$ matched that of Fig. 682 2 , yielding $W_{\mathrm{sc}}=45.69 \mathrm{eV}$. The difference between this 683 value of $W_{\mathrm{sc}}$ and that calculated in Sec. 2 was used to as- 684 sign an additional systematic uncertainty of $15 \mathrm{eV}$ (see 685 Eq. 5). For all recoil energies, the nuclear recoil yields 686 are set to be equal to the electronic recoil yields mul- 687 tiplied by a quenching factor $\alpha$, where we have chosen 688 for S1 $\alpha_{\mathrm{S} 1}=0.53$ and for S2 $\alpha_{\mathrm{S} 2}=0.17$, corresponding 689 to nuclear recoil yields of $Y_{1}=\alpha_{\mathrm{S} 1} / W_{\mathrm{sc}}=11.6 \mathrm{ph} / \mathrm{keV} 690$ for primary scintillation and $Y_{2}=\alpha_{\mathrm{S} 2} / W_{\mathrm{i}}=6.9 \mathrm{e}^{-} / \mathrm{keV} 691$ for ionization.

The Monte Carlo included the pressure vessel, PTFE walls of the field cage and PTFE reflector, PMT array, mesh grids, and lead block. A detailed model for the wavelength shifting introduced by the TPB was not implemented, however the reflectivities on the walls and the PTFE reflector were selected such that the zdependence of S1 matched that of Fig. 2 in a simulation using axially-incident $662 \mathrm{keV}$ gamma rays, similar to the experimental setup using the ${ }^{137} \mathrm{Cs}$ source described in Sec. 2. The walls were taken to be $100 \%$ reflective and the back reflector to be $79 \%$ reflective. Though these values are not consistent with the expected PTFE reflectivity in xenon gas (from [24] expected to be 50$60 \%$ ), they properly reproduced the linear geometric dependence of S1. The source was modeled by emitting neutrons from a single point behind the lead block in a random direction, accompanied by a $4.4 \mathrm{MeV}$ gamma ray also emitted in a random direction. One neutron and 

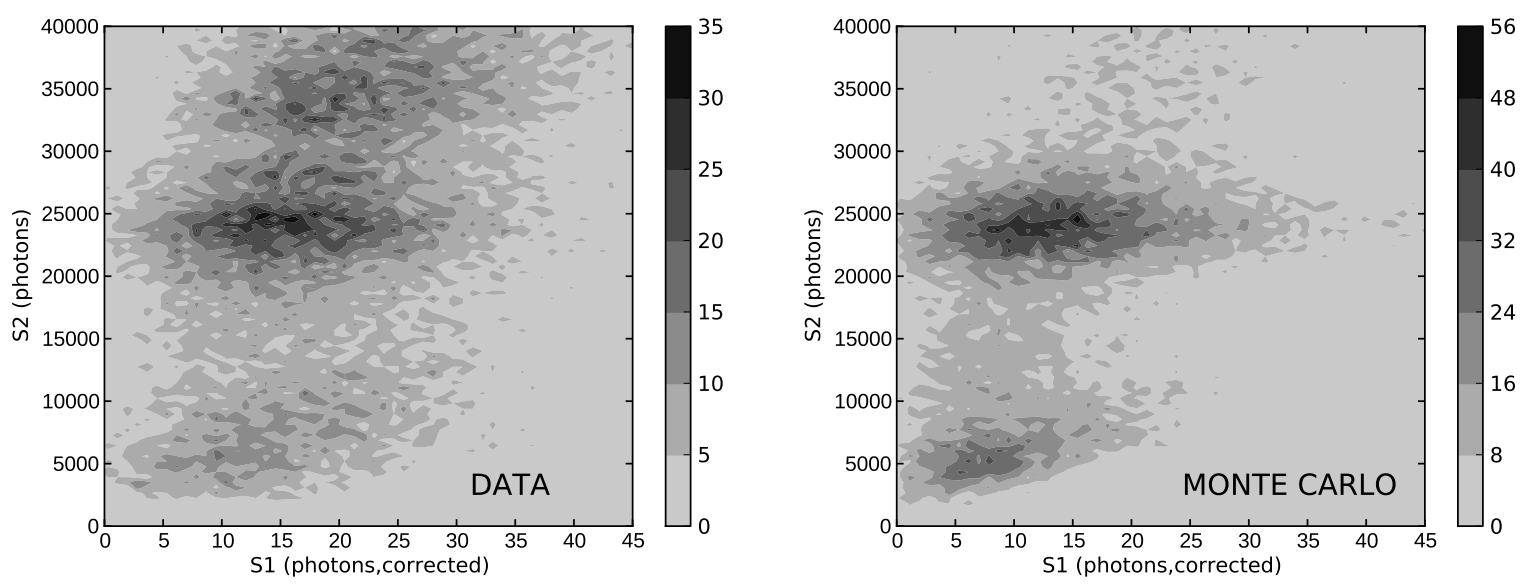

Figure 11: S2 vs. S1 for low-energy events in experiment and Monte Carlo simulations generated based on the selected constant nuclear recoil yields. Note the presence of the nuclear recoil band at low S2 in both distributions. The band at S2 35000 produced due to $40 \mathrm{keV}$ gammas emitted in neutron inelastic scattering on ${ }^{129} \mathrm{Xe}$ nuclei is present in the experimental distribution due to low-energy neutron events (for which the neutron- ${ }^{129} \mathrm{Xe}$ inelastic scattering cross section is higher) not included in the Monte Carlo simulation.

one gamma ray were emitted per event, and the spec- 728 trum of emitted neutron energies was taken to be that 729 calculated for neutrons produced with a carbon nucleus 730 in the first excited state ${ }^{12} \mathrm{C}^{*}$ ( similar to that shown in 731 Fig. 3). The neutron interactions were modeled using 732 the Geant4 high-precision (HP) neutron models (Geant ${ }_{733}$ version 4.9.3.p02 was used with G4NDL3.13), which 734 use neutron cross section data from evaluated nuclear 735 data libraries. The consistency of the Monte Carlo and 736 calculated spectra of nuclear recoils can be seen by ex- 737 amining Fig. A.14.

In the simulation, secondary electrons produced by 739 high-energy photons deposited their energy in steps of 740 maximum length $1 \mathrm{~mm}$, and for each step a cluster of 741 ionization electrons was produced containing a number 742 of electrons generated according to the energy deposited 743 in the step. The final location of each ionization electron 744 after drifting through the active region was calculated, 745 taking into account diffusion. The electroluminescent 746 process was modeled by producing a number of photons 747 equal to the EL gain $g$ at the arrival location of each ion- 748 ization electron at the EL plane. Individual EL photons 749 were not tracked individually but their detection proba- 750 bilities were determined via a look-up table indexed by 751 $(x, y)$ production location in the 2D EL plane. This ta- 752 ble was produced in an independent Monte Carlo run in 753 which $10^{6}$ photons were generated per point on a grid 754 dividing the EL plane and the detection probability for 755 each PMT recorded based on the number of photons 756 collected out of the $10^{6}$ generated. Each photon pro- 757 duced via primary scintillation was tracked throughout 758 its entire trajectory. From the record of photons detected 759 at each time in the event, realistic waveforms were constructed for each PMT matching the noise characteristics of the experimental waveforms and adding exponential pulses for each photoelectron detected. The resulting waveforms were passed through a nearly identical analysis to that of the experimental data to give results that could be compared directly to experiment. To simulate the experimental gamma-ray tagging procedure, only neutron-induced events, those in which a neutron scattered inelastically on any material in the simulation or elastically on xenon, were considered in the analysis. To roughly match the detector threshold effects observed at low $\mathrm{S} 1$ values in experiment, a peakfinding threshold was chosen appropriately in the Monte Carlo analysis.

Figure 11 shows the $\mathrm{S} 2$ signals plotted against $\mathrm{S} 1$ corrected for Z-dependence in both data and Monte Carlo. Additional Gaussian smearing was applied to the S1 and S2 values determined in Monte Carlo to account for inaccuracies in modeling the experimental energy resolution, due to details such as geometric dependencies in the detection procedure. The analysis included tagging, single-pulse, diffusion, and radial cuts. Similar cuts were made in the corresponding Monte Carlo run, and the good qualitative agreement, particularly in the location of the nuclear recoil band, shows that the nuclear recoil quenching factors employed in the simulation are reasonable. In particular, for events contained inside the region corresponding to the nuclear recoil band (as defined in figure 9), the mean S1 values for data (Monte Carlo) are 19.68 (15.32) with standard deviations of 13.21 (13.58), and the mean S2 values 
for data (Monte Carlo) are 9230 (8803) with standard 810 deviation 3791 (3752). One significant discrepancy in 811 the Monte Carlo results is the relatively fewer number ${ }_{812}$ of events in the band lying at approximately $35000 \mathrm{~S} 2{ }_{813}$ photons which is due to $40 \mathrm{keV}$ gamma rays produced 814 by neutron inelastic scattering on ${ }^{129} \mathrm{Xe}$. This is due to ${ }_{815}$ the fact that only neutrons accompanied by a $4.4 \mathrm{MeV}{ }_{816}$ gamma (energies 2-6 MeV) are considered in the Monte ${ }_{817}$ Carlo, while in the experimental data some fraction of ${ }_{818}$ the neutrons accompanied by the $7.7 \mathrm{MeV}$ gamma (en- ${ }_{819}$ ergies $<3 \mathrm{MeV}$ ) are also included. This is because the ${ }_{820}$ $7.7 \mathrm{MeV}$ gamma may not fully deposit its energy in the ${ }_{821}$ $\mathrm{NaI}$ scintillator and therefore may deposit an energy in 822 the window of allowed $\mathrm{NaI}$ energies $\left(2.4-5.0 \mathrm{MeV}\right.$ for ${ }_{223}$ the experimental run described in this section, and sim- 824 ilar for that described in Sec. 3.1 - 3.3). The inelastic 825 $\mathrm{n}+{ }^{129} \mathrm{Xe}$ scattering cross section is significantly higher ${ }_{826}$ [38] for these lower energy neutrons, so one should ex- 827 pect relatively more $40 \mathrm{keV}$ gamma rays in the experi- 828 mental data. However, the nuclear recoils produced by ${ }_{829}$ these lower energy neutrons will be lower in energy and 830 therefore should not contribute significantly to the nu- 831 clear recoil spectrum at the energies observable in the ${ }_{832}$ data.

\section{Conclusions}

We have simultaneously observed ionization and scintillation produced by nuclear recoils in gaseous xenon. It was confirmed that xenon in the gas phase, as in the liquid phase, is capable of distinguishing nuclear and electronic recoils based on the ratio of observed scintillation to ionization. It appears that relative to the corresponding yields for electronic recoils, the primary scintillation yield for nuclear recoils is quenched by a factor of roughly 2 , and the ionization yield by a factor of roughly 5. Further investigation is required to determine precise nuclear recoil yields and fully investigate the potential advantages offered by gas phase oper- 843 ation. The use of a monoenergetic source of neutrons in 844 future studies would avoid the complexities introduced 845 by having to match experimental results with a recoil 846 spectrum calculated from a source spectrum known to 847 a significant degree of uncertainty. The greatest impact 848 of the present results is the ability to provide a starting ${ }_{849}$ point for such future measurements of nuclear recoils in 850 gaseous xenon. In addition, should gaseous xenon be 851 found capable of determining the direction of a nuclear recoil, such studies will become even more important.

The presence of gamma-induced energy depositions in regions from which S2 cannot be collected provides an evident background and is known to be of concern also in the liquid phase. This is likely to be of even greater concern in the gas phase, as gamma rays can travel farther, and is evident in the inability to eliminate events consisting only of a single fluorescent $x$-ray. In such events, $\mathrm{x}$-rays produced outside of the active region were able to travel into the active region and interact there. Such events can in principle be eliminated with a fiducial cut, but this would require a larger detector than the one used in the present experiment.

Though only estimates of the nuclear recoil yields were given, they can be used to predict the necessary light collection efficiency required to observe recoils of a given energy of interest. With 3\% light collection efficiency at the EL plane, we were not able to see many recoils with energy less than approximately $30 \mathrm{keV}$. Therefore a $\sim 10 \%$ light collection efficiency at least would be necessary to perform a stronger measurement of the nuclear recoil yields down to near $10 \mathrm{keV}$. The higher photon statistics will also help in characterizing the electronic/nuclear recoil discrimination power based on $\mathrm{S} 2 / \mathrm{S} 1$ that is possible in the gas phase. In this study, the TPB was found to be a necessity for achieving enough light collection efficiency to identify the nuclear recoils despite the complications it introduced. In the future more sophisticated ideas such as the use of light guides coupled to PMTs [15] may be necessary to achieve the light collection regime of interest.

\section{Appendix A. Radioisotope Neutron Sources}

\section{Appendix A.1. Neutron Production Mechanism}

The neutron sources used in this study all consist of an $\alpha$-emitting radioactive isotope mixed with beryllium $\left({ }^{9} \mathrm{Be}\right)$ and generate neutrons based on the $(\alpha, \mathrm{n})$ reaction [39]

$$
\alpha+{ }^{9} \mathrm{Be} \rightarrow{ }^{12} \mathrm{C}+\mathrm{n} .
$$

The Q-value of the reaction is $\mathrm{Q}=5.701 \mathrm{MeV}$, and this energy is released in the form of neutron kinetic energy, neglecting any carbon recoil kinetic energy, unless the carbon nucleus is left in an excited state, in which case some of the energy is emitted in the form of a gamma ray in coincidence with the neutron. ${ }^{5}$ If left in the first excited state ${ }^{12} \mathrm{C}^{*}$, a gamma ray of energy $4.439 \mathrm{MeV}$ is emitted, and if left in the second excited state ${ }^{12} \mathrm{C}^{* *}$, a gamma ray of energy $7.654 \mathrm{MeV}$

\footnotetext{
${ }^{5}$ The mean lifetimes of the excited carbon nuclear states of interest here are so short $(<0.1 \mathrm{ps})$ compared to the measurable time scale that the gamma can be considered to be emitted simultaneously with the neutron for practical purposes.
} 
Table A.1: Selected radioactive decay products of ${ }^{238} \mathrm{Pu}$ and ${ }^{241} \mathrm{Am}$. All data shown is from [27]. Both isotopes emit alpha particles with an average energy of approximately 5.5 MeV. Gamma ray emission from 88 ${ }^{238} \mathrm{Pu}$ is negligible, while some low-energy gamma rays are emitted 889 by ${ }^{241} \mathrm{Am}$. In particular, the $60 \mathrm{keV}$ gamma ray is likely to produce significant background without sufficient shielding of the source.

\begin{tabular}{lccc} 
Isotope, $\tau_{1 / 2}, \mathrm{Q}(\mathrm{keV})$ & Prod. & $\mathrm{E}(\mathrm{keV})$ & $\% \mathrm{BR}$ \\
\hline${ }^{238} \mathrm{Pu}$ & $\alpha$ & 5357.7 & 0.105 \\
$\tau_{1 / 2}=87.7 \pm 0.3 \mathrm{yr}$ & $\alpha$ & 5456.3 & 28.98 \\
$\mathrm{Q}=5593.20 \pm 0.19$ & $\alpha$ & 5499.03 & 70.91 \\
\hline${ }^{241} \mathrm{Am}$ & $\gamma$ & 13.946 & 9.6 \\
$\tau_{1 / 2}=432.2 \pm 0.7 \mathrm{yr}$ & $\gamma$ & 59.5412 & 35.9 \\
$\mathrm{Q}=5631.81 \pm 0.12$ & $\alpha$ & 5388.23 & 1.6 \\
& $\alpha$ & 5442.80 & 13.0 \\
& $\alpha$ & 5485.56 & 84.5
\end{tabular}

is emitted [40]. In this study we detect the coincident gamma and include it in the acquisition trigger to tag neutron-emitting decays and thereby significantly reduce the number of background events acquired.

We describe here how to calculate the neutron spectra of radioisotope sources under the assumption that their active regions consist of a uniform volume of ${ }^{9} \mathrm{Be}$ throughout which the $\alpha$-emitting isotope is uniformly distributed. We also assume that the total number of $\alpha$-emitting isotope atoms present is much less than the total number of beryllium atoms in the mixture, so that each emitted $\alpha$ can be considered to interact with only 900 atoms of beryllium. In addition, we do not consider 901 neutrons produced due to the break-up reaction $\alpha+{ }^{9} \mathrm{Be}{ }^{902}$ $\rightarrow \alpha^{\prime}+{ }^{9} \mathrm{Be}^{*},{ }^{9} \mathrm{Be}^{*} \rightarrow{ }^{8} \mathrm{Be}+\mathrm{n}$ [41]. These neutrons lie ${ }^{903}$ at lower energies $\lesssim 3 \mathrm{MeV}$, and they will not be ob- 904 served in the adopted trigger scheme as no coincident 905 gamma ray is emitted. Our calculations follow those ${ }^{906}$ of [41], [42], and [43], and make use of $(\alpha, \mathrm{n})$ cross ${ }^{907}$ sections from the Japanese Evaluated Nuclear Data Li- 908 brary (JENDL) [44] and neutron-xenon scattering cross sections from the Evaluated Nuclear Data File (ENDF) [38]. The cross sections were processed using the tools developed in [45]. Table A.1 gives information on the decays of isotopes ${ }^{238} \mathrm{Pu}$ and ${ }^{241} \mathrm{Am}$ used as $\alpha$-emitters in the sources used in this study.

\section{Appendix A.2. Spectrum of Emitted Neutrons}

The neutron production process proceeds as follows. 9 The $\alpha$-emitting isotope decays, yielding an $\alpha$ particle of mass $m_{\alpha}$ that travels through the surrounding medium of beryllium atoms of mass $m_{b}$, losing energy and often stopping completely without undergoing the reac- 912 tion shown in Eq. A.1. However, some alpha particles ${ }_{913}$ (of order 1 in $10^{4}$ ) will undergo the neutron-producing 914 896 897

reaction of interest at an energy of $E_{\alpha}$ and generate a neutron of mass $m_{n}$ at some angle $\theta$ in the center of mass frame of the interaction that can be directly related to the emitted neutron energy $E_{n}$ via [42]

$$
\begin{aligned}
\cos \theta= & E_{n}\left(m_{n}+m_{c}\right)^{2}-E_{\alpha}\left(m_{b} m_{c}+m_{n} m_{\alpha}\right) \\
& \frac{-Q^{\prime} m_{c}\left(m_{n}+m_{c}\right)}{2\left[E_{\alpha} m_{\alpha} m_{n}\left(E_{\alpha} m_{b} m_{c}+Q^{\prime} m_{c}\left(m_{n}+m_{c}\right)\right)\right]^{1 / 2}},
\end{aligned}
$$
899

$$
\begin{aligned}
& G\left(E_{n} ; E_{\alpha}\right) d E_{n}= \\
& \quad \frac{1}{\sigma_{T}\left(E_{\alpha}\right)} \frac{d \sigma\left(E_{n} ; E_{\alpha}\right)}{d \Omega} \cdot \frac{4 \pi}{E_{n}(\theta=0)-E_{n}(\theta=\pi)} d E_{n},
\end{aligned}
$$

where $\sigma_{T}\left(E_{\alpha}\right)$ is the total cross section of the $(\alpha, \mathrm{n})$ reaction for alpha energy $E_{\alpha}$, shown in Fig. A.12, and $d \sigma / d \Omega$ is the differential cross section. The number of neutrons with energy $E_{n}$ produced for emitted alpha $i$ of energy $E_{\alpha, i}$ can then be determined by multiplying $G\left(E_{n} ; E_{\alpha}\right)$ by the probability of interaction at alpha energy $E_{\alpha}$ along the track, that is, $\rho \sigma_{T}\left(E_{\alpha}\right) d x=$ $\rho \sigma_{T}\left(E_{\alpha}\right)\left(d x / d E_{\alpha}\right) d E_{\alpha}$, and integrating over all energies from 0 to $E_{\alpha, i}$,

$$
\begin{aligned}
& N_{i}\left(E_{n}\right)= \\
& \quad \int_{0}^{E_{\alpha, i}} \frac{4 \pi\left[d \sigma\left(E_{n} ; E_{\alpha}\right) / d \Omega\right]}{d E_{\alpha} /(\rho d x)\left[E_{n}(\theta=0)-E_{n}(\theta=\pi)\right]} d E_{\alpha} .
\end{aligned}
$$

The final spectrum will be a sum of such integrals over the emitted $\alpha$ energies of the source weighted by their branching ratios $x_{i}$,

$$
N\left(E_{n}\right)=\sum_{i} x_{i} N_{i}\left(E_{n}\right) .
$$

Figure 3 of Sec. 3.1 shows the spectrum for a ${ }^{241} \mathrm{Am} / \mathrm{Be}$ source calculated assuming the alpha particle energies and branching ratios from Table A.1. Note that 


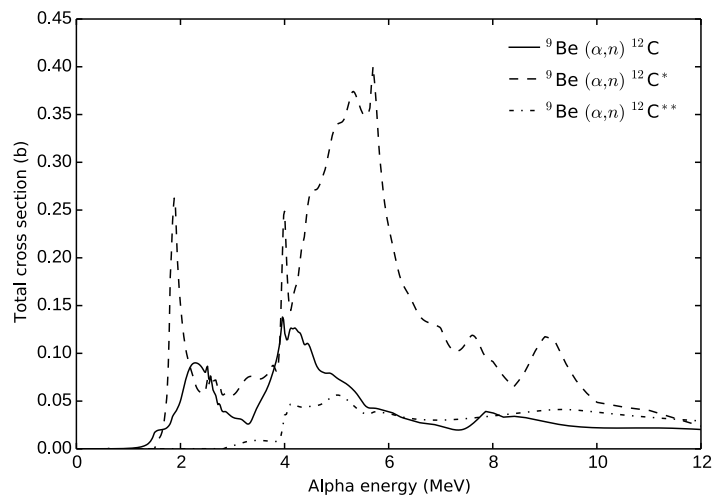

Figure A.12: Total cross section for the reaction ${ }^{9} \mathrm{Be}(\alpha, \mathrm{n}){ }^{12} \mathrm{C}$ for which the product ${ }^{12} \mathrm{C}$ nucleus was left in the ground state (solid line), first excited state (dashed line), and second excited state (dot-dashed line). All data shown in this figure are from JENDL [44]. this calculation is only valid for $d x \ll \lambda_{\alpha, \mathrm{n}}$, where $\lambda_{\alpha, \mathrm{n}}$ is the mean interaction length for the $(\alpha, \mathrm{n})$. However, the average length of the track produced by a $5.5 \mathrm{MeV}$ alpha particle in beryllium (density $\rho=1.23 \times 10^{23} \mathrm{~cm}^{-3}[46]$ ) can be calculated using the alpha stopping power from [47] as $\int(d x / d E) d E \approx 28 \mu \mathrm{m}$. Using total cross section $\sigma_{T}<0.4$ barns (see Fig. A.12), $\lambda_{\alpha, \mathrm{n}}=\left(\rho \sigma_{T}\right)^{-1}>20$ $\mathrm{cm}$, and therefore this condition holds.

\section{Appendix A.3. Resulting Spectrum of Nuclear Recoils}

From a spectrum of emitted neutrons, one can construct a spectrum of nuclear recoils produced when those neutrons are incident on a volume of xenon atoms by knowing the cross section for neutron elastic scattering (differential with respect to solid angle). Similar to Eq. A.2, we can express the energy of a nuclear recoil in terms of the cosine of the scattering angle in the centerof-mass frame of the neutron-nucleus collision as

$$
E_{x^{\prime}}=\frac{2 E_{n} m_{n} m_{x, a}}{\left(m_{n}+m_{x, a}\right)^{2}}(1-\cos \theta)
$$

where $m_{x, a}$ is the mass of the target xenon nucleus and $a$ is an index corresponding to the isotope of the nucleus.

Similar to Eq. A.3, we can use this relation to write the angular distribution of scattered neutrons in terms of the recoil energy, and determine the number of neutron interactions per unit recoil energy $N_{r, a}\left(E_{x^{\prime}} ; E_{n}\right)$ yielding a nuclear recoil with energy in the interval $\left(E_{x^{\prime}}, E_{x^{\prime}}+\right.$ $\left.d E_{x^{\prime}}\right)$ for a neutron of energy $E_{n}$ incident on a xenon nucleus of isotope $a$,
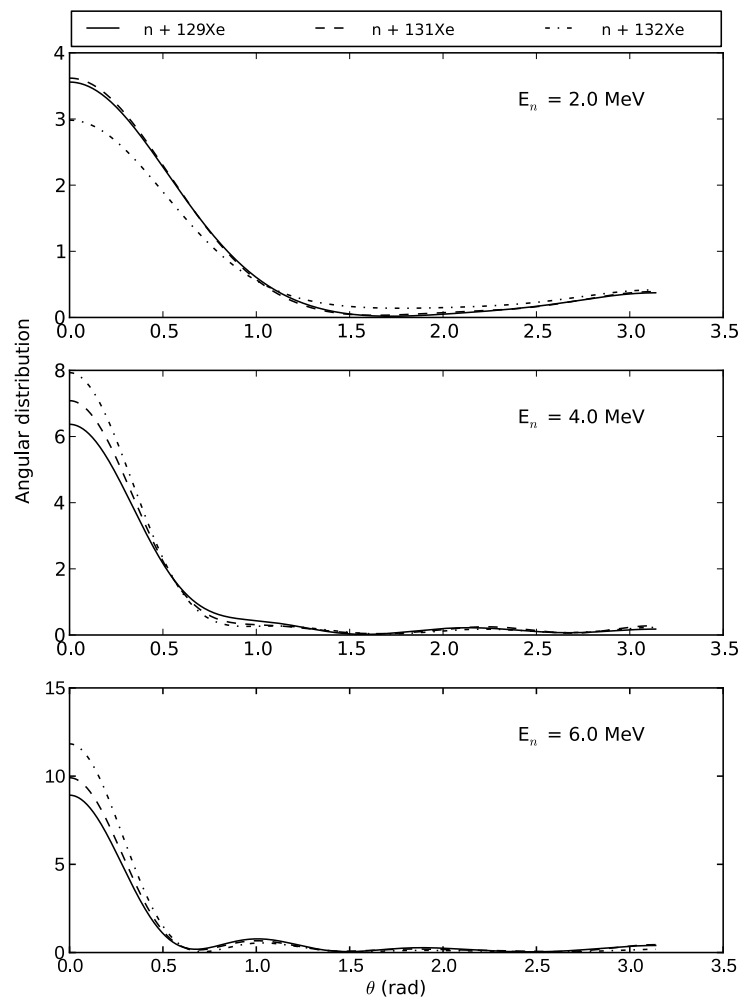

Figure A.13: Angular distributions (data from [38]) for elastic neutron scattering, $\mathrm{n}+\mathrm{Xe} \rightarrow \mathrm{n}^{\prime}+\mathrm{Xe}^{\prime}$, for three different neutron energies incident on three different xenon isotopes. Note the strong peak in the forward direction, followed by one or several peaks at higher angles which are responsible for the structure in the nuclear recoil spectrum at higher energies.

$$
\begin{aligned}
& N_{r, a}\left(E_{x^{\prime}} ; E_{n}\right)= \\
& \quad \frac{\left(m_{n}+m_{x, a}\right)^{2}}{m_{n} m_{x, a}} \cdot \frac{\pi}{E_{n}}\left[\frac{d \sigma\left(E_{x^{\prime}} ; E_{n}\right)}{d \Omega}\right]_{a} \rho \Delta x,
\end{aligned}
$$

where $\rho$ is the density of the xenon volume and $\Delta x$ is the thickness of xenon traversed by the neutron.

Figure A.13 shows the angular distribution for neutrons scattered off of several different xenon isotopes. Note that because we will be interested in the normalized spectrum, and $\rho \Delta x$ is a constant independent of the isotope, it can be absorbed into an overall normalization factor in the final spectrum, which is a weighted superposition of individual nuclear recoil spectra summed over xenon isotopes and integrated over neutron energies,

$$
N\left(E_{x^{\prime}}\right)=\sum_{a} f_{a} \int_{0}^{\infty} N\left(E_{n}\right) N_{r, a}\left(E_{x^{\prime}} ; E_{n}\right) d E_{n}
$$


where the $f_{a}$ are the fractional compositions of natural 977 xenon for each isotope and $N\left(E_{n}\right)$ is the spectrum of 978 emitted neutrons from Eq. A.5.

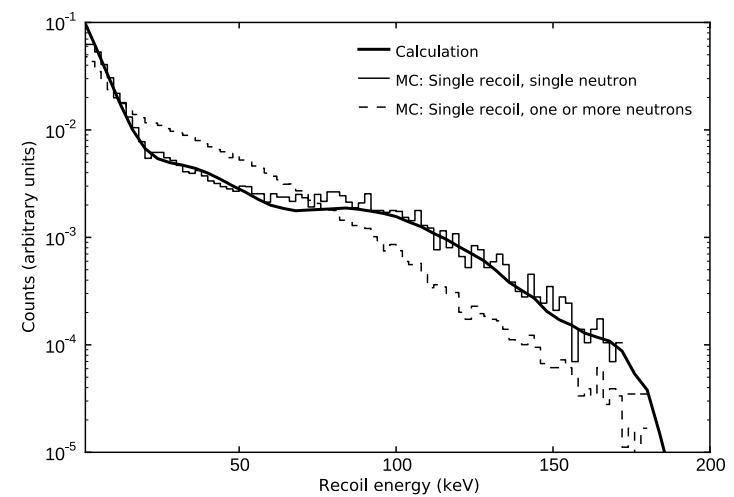
238 a ${ }^{238} \mathrm{Pu} / \mathrm{Be}$ source in which the carbon nucleus resulting from the ${ }^{9} \mathrm{Be}(\alpha, \mathrm{n}){ }^{12} \mathrm{C}$ reaction was left in the first excited state. The spectrum 995 was obtained from a calculation using Eq. A.7 and Eq. A.8 (thick solid 996 line) and from a Geant4 Monte Carlo. Two curves are shown from the 997 same Monte Carlo run. The solid line is the spectrum considering only 998 events containing a single xenon nuclear recoil and a single neutron. 999 The dashed line is the spectrum of events containing a single xenon 1000 nuclear recoil, but in which the event may have contained multiple ${ }^{1001}$ neutrons produced by interaction of the incident neutron with the lead. ${ }^{1002}$ The presence of these secondary neutrons significantly distorts the ${ }^{1003}$ spectrum of xenon nuclear recoils.

Office of Basic Energy Sciences, of the U.S. Department of Energy, and the National Energy Research Scientific Computing Center (NERSC), supported by the Office of Science of the U.S. Department of Energy, both under Contract No. DE-AC02-05CH11231; the European Research Council under the Advanced Grant 339787-NEXT; the Ministerio de Economía y Competitividad of Spain under Grants CONSOLIDER-Ingenio 2010 CSD2008-0037 (CUP), FPA2009-13697-C0404, FPA2009-13697-C04-01, FIS2012-37947-C04-01, FIS2012-37947-C04-02, FIS2012-37947-C04-03, and FIS2012-37947-C04-04; and the Portuguese FCT and FEDER through the program COMPETE, Projects PTDC/FIS/103860/2008 and PTDC/FIS/112272/2009.

J. Renner acknowledges the support of a Department of Energy National Nuclear Security Administration Stewardship Science Graduate Fellowship, grant number DE-FC52-08NA28752.

[1] E. Aprile, A. E. Bolotnikov, A. I. Bolozdynya, T. Doke, Noble Gas Detectors, Wiley VCH, Verlag GmbH \& Co. KGaA, Weinheim, 2006.

[2] E. Aprile, T. Doke, Liquid xenon detectors for particle physics and astrophysics, Rev. Mod. Phys. 82 (2010) 2053-2097.

[3] D. S. Akerib, et al. (LUX Collaboration), First Results from the LUX Dark Matter Experiment at the Sanford Underground Research Facility, Phys. Rev. Lett. 112 (2014) 091303.

[4] E. Aprile, et al. (XENON100 Collaboration), Dark Matter Results from 225 Live Days of XENON100 Data, Phys. Rev. Lett. 109 (2012) 181301

[5] D.Yu. Akimov, et al. (ZEPLINIII Collaboration), WIMPnucleon cross-section results from the second science run of ZEPLIN-III, Phys. Lett. B 709 (2012) 14-20.

[6] M. Auger, et al. (EXO Collaboration), Search for Neutrinoless Double-Beta Decay in Xe136 with EXO-200, Phys. Rev. Lett. 109 (2012) 032505.

[7] G. Bertone, D. Hooper, J. Silk, Particle dark matter: evidence, candidates and constraints, Physics Reports 405 (2005) 279390.

[8] J. J. Gómez-Cadenas, J. Martín-Albo, M. Mezzetto, F. Monrabal, M. Sorel, The search for neutrinoless double beta decay, Riv. Nuovo Cim. 35 (2012) 29-98.

[9] M. Szydagis, N. Barry, K. Kazkaz, J. Mock, D. Stolp, M. Sweany, M. Tripathi, S. Uvarov, N. Walsh, M. Woods, NEST: a comprehensive model for scintillation yield in liquid xenon, JINST 6 (2011) P10002, http://nest.physics.ucdavis.edu/site.

[10] E. Aprile, K. L. Giboni, P. Majewski, K. Ni, M. Yamashita, Observation of anticorrelation between scintillation and ionization for MeV gamma rays in liquid xenon, Phys. Rev. B 76 (2007) 014115 .

[11] P. Sorensen, et al., The scintillation and ionization yield of liquid xenon for nuclear recoils, Nucl. Instrum. Methods A 601 (2009) 339-346.

[12] M. Horn, et al. (ZEPLINIII Collaboration), Nuclear recoil scintillation and ionisation yields in liquid xenon from ZEPLIN-III data, Phys. Lett. B 705 (2011) 471-476.

[13] A. Bolotnikov, B. Ramsey, The spectroscopic properties of highpressure xenon, Nucl. Instrum. Methods A 396 (1997) 360-370.

[14] E. Conti, et al., Correlated fluctuations between luminescence and ionization in liquid xenon, Phys. Rev. B 68 (2003) 054201. 
[15] D. R. Nygren, Columnar recombination: a tool for nuclear recoil 1102 directional sensitivity in a xenon-based direct detection WIMP 1103 search, J. Phys. Conf. Ser. 460 (2013) 012006.

[16] D. C. Herrera, et al., Study of Columnar Recombination in 1105 Xe+trymethylamine Mixtures using a Micromegas-TPC, Pro- 1106 ceedings of Science TIPP2014 (2014) 054.

[17] J. Renner, Ph.D. thesis, University of California, Berkeley 1108 (2014).

[18] J. Renner, V. Gehman, A. Goldschmidt, C. Oliveira, D. Nygren, 1110 Characterization of Nuclear Recoils in High Pressure Xenon 1111 Gas: Towards a Simultaneous Search for WIMP Dark Mat- 1112 ter and Neutrinoless Double Beta Decay, Physics Procedia 611113 (2015) 766-773.

[19] J. White, Presentation at PPC 2008: 2nd International Workshop 1115 on Interconnection Between Particle Physics and Cosmology. 1116

[20] V. Álvarez, et al. (NEXT Collaboration), Near-intrinsic energy 1117 resolution for 30-662 keV gamma rays in a high pressure xenon 1118 electroluminescent TPC, Nucl. Instrum. Methods A 708 (2013) 1119 $101-114$.

1120

21] D. Nygren, High-pressure xenon gas electroluminescent TPC 1121 for $0-v \beta \beta$-decay search, Nucl. Instrum. Methods A 603 (2009) 1122 337-348.

22] C. M. B. Monteiro, L. M. P. Fernandes, J. A. M. Lopes, L. C. 1124 C. Coelho, J. F. C. A. Veloso, J. M. F. dos Santos, K. Giboni, 1125 E. Aprile, Secondary scintillation yield in pure xenon, JINST 21126 (2007) P05001.

[23] V. Gehman, S. Seibert, K. Rielage, A. Hime, Y. Sun, D.-M. Mei, 1128 J. Maassen, D. Moore, Fluorescence efficiency and visible re- 1129 emission spectrum of tetraphenyl butadiene films at extreme ul- 1130 traviolet wavelengths, Nucl. Instrum. Meth. A 654 (2011) 116- 1131 121.

[24] C. Silva, J. Pinto da Cunha, A. Pereira, V. Chepel, M. I. Lopes, V. Solovov, F. Neves, Reflectance of polytetrafluoroethylene for xenon scintillation light, J. Appl. Phys. 107 (2010) 064902.

[25] ROOT, http://root.cern.ch/drupal.

[26] FMWK, https://cdcvs.fnal.gov/redmine/projects/nusoft/wiki/ FMWK.

[27] L. P. Ekström, R. B. Firestone, WWW Table of Radioactive Isotopes, database version 2/28/99, http://ie.lbl.gov/toi.

[28] A. Parsons, T. Edberg, B. Sadoulet, S. Weiss, J. Wilkerson, K. Hurley, R. Lin, G. Smith, High pressure gas scintillation drift chambers with wave-shifter fiber readout, IEEE Trans. Nucl. Sci. 37 (1990) 541-546.

[29] S. J. C. do Carmo, F. I. G. M. Borges, F. P. Santos, T. H. V. T. Dias, C. A. N. Conde, Absolute primary scintillation yield of gaseous xenon under low drift electric fields for $5.9 \mathrm{keV} \mathrm{X}$-rays, JINST 3 (2008) P07004.

[30] R. L. Platzman, Total Ionization in Gases by High-Energy Particles: An Appraisal of Our Understanding, Int. J. Appl. Rad. Iso. 10 (1961) 116-127.

[31] J. Mock, N. Barry, K. Kazkaz, D. Stolp, M. Szydagis, M. Tripathi, S. Uvarov, M. Woods, N. Walsh, Modeling pulse characteristics in xenon with NEST, JINST 9 (2014) T04002.

[32] V. Álvarez, et al., Ionization and scintillation response of highpressure xenon gas to alpha particles, JINST 8 (2013) P05025.

[33] C. E. Dahl, Ph.D. thesis, Princeton University (September 2009).

[34] S. F. Biagi, Monte Carlo simulation of electron drift and diffusion in counting gases under the influence of electric and magnetic fields, Nucl. Instrum. Methods A 421 (1999) 234-240.

[35] D. S. Akerib, et al. (LUX Collaboration), The Large Underground Xenon (LUX) experiment, Nucl. Instrum. Meth. A 704 (2013) 111-126.

[36] E. Aprile, et al. (XENON100 Collaboration), Analysis of the XENON100 dark matter search data, Astropart. Phys. 54 (2014)
$11-24$.

[37] S. Agostinelli, et al., Geant4-a simulation toolkit, Nucl. Instrum. Methods A 506 (2003) 250-303.

[38] Evaluated Nuclear Data File (ENDF), http://www.nndc.bnl.gov/exfor/endf00.jsp.

[39] G. F. Knoll, Radiation Detection and Measurement, 3rd Edition, Wiley, Hoboken, N.J., 2000.

[40] Evaluated Nuclear Structure Data File (ENSDF), http://www.nndc.bnl.gov/ensdf.

[41] K. Geiger, L. van der Zwan, Radioactive neutron source spectra from ${ }^{9} \operatorname{Be}(\alpha, \mathrm{n})$ cross section data, Nucl. Instrum. Methods 131 (1975) 315-321.

[42] A. Vijaya, A. Kumar, The neutron spectrum of Am-Be neutron sources, Nucl. Instrum. Methods 111 (1973) 435-440.

[43] A. Kumar, P. Nagarajan, Neutron spectra of ${ }^{239} \mathrm{Pu}-\mathrm{Be}$ neutron sources, Nucl. Instrum. Methods 140 (1977) 175-179.

[44] T. Murata, H. Matsunobu, K. Shibata, JENDL $(\alpha, n)$ Reaction Data File 2005, http://wwwndc.jaea.go.jp/ftpnd/jendl/jendl-an2005.html.

[45] C. M. Mattoon, B. R. Beck, N. R. Patel, N. C. Summers, G. W. Hedstrom, D. A. Brown, Generalized Nuclear Data: A New Structure (with Supporting Infrastructure) for Handling Nuclear Data, Nuclear Data Sheets 113 (2012) 3145-3171, https://ndclx4.bnl.gov/gf/project/gnd.

[46] J. Beringer, et al., Review of Particle Physics, Phys. Rev. D 86 (2012) 010001

[47] NIST, ASTAR: stopping power and range tables for helium ions, http://physics.nist.gov/PhysRefData/Star/Text/ASTAR.html. Accessed July 19, 2013.

[48] P. D. Biévre, P. Taylor, Table of the isotopic compositions of the elements, Int. J. Mass Spec. Ion Processes 123 (1993) 149-166. 